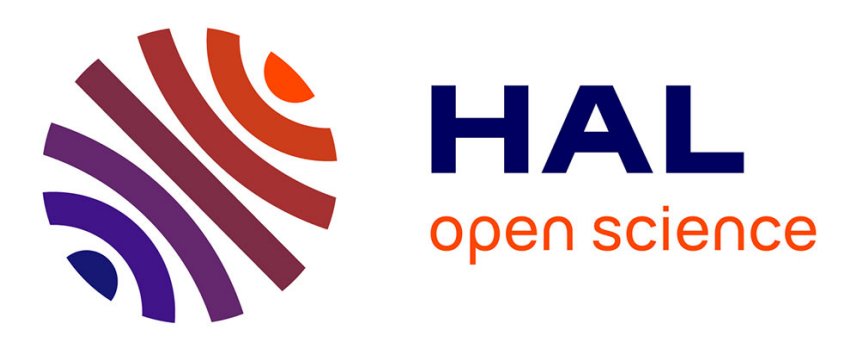

\title{
Upwind Residual discretization of enhanced Boussinesq equations for wave propagation over complex bathymetries
}

\author{
Mario Ricchiuto, Andrea Gilberto Filippini
}

\section{- To cite this version:}

Mario Ricchiuto, Andrea Gilberto Filippini. Upwind Residual discretization of enhanced Boussinesq equations for wave propagation over complex bathymetries. [Research Report] RR-8311, INRIA. 2013. hal-00826912

\section{HAL Id: hal-00826912 \\ https://hal.inria.fr/hal-00826912}

Submitted on 7 Jun 2013

HAL is a multi-disciplinary open access archive for the deposit and dissemination of scientific research documents, whether they are published or not. The documents may come from teaching and research institutions in France or abroad, or from public or private research centers.
L'archive ouverte pluridisciplinaire HAL, est destinée au dépôt et à la diffusion de documents scientifiques de niveau recherche, publiés ou non, émanant des établissements d'enseignement et de recherche français ou étrangers, des laboratoires publics ou privés. 


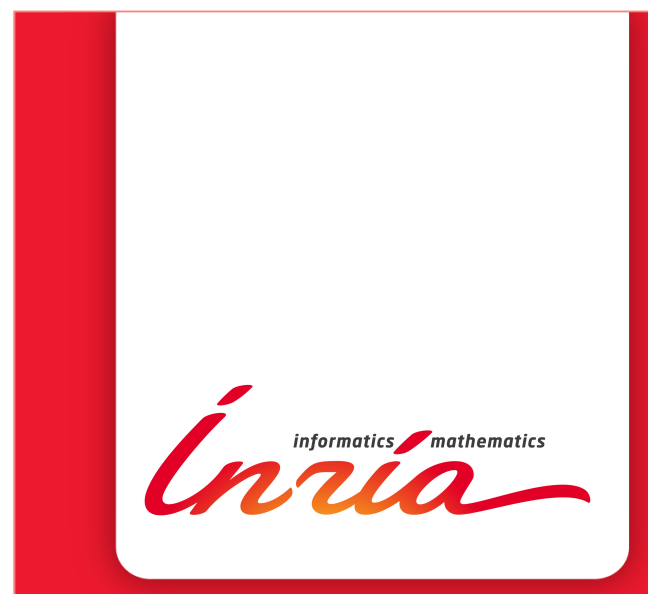

\section{Upwind Residual discretization of} enhanced Boussinesq equations for wave propagation over complex bathymetries

M. Ricchiuto, A.G. Filippini

RESEARCH REPORT

$\mathrm{N}^{\circ} 8311$

May 2013

Project-Teams BACCHUS 



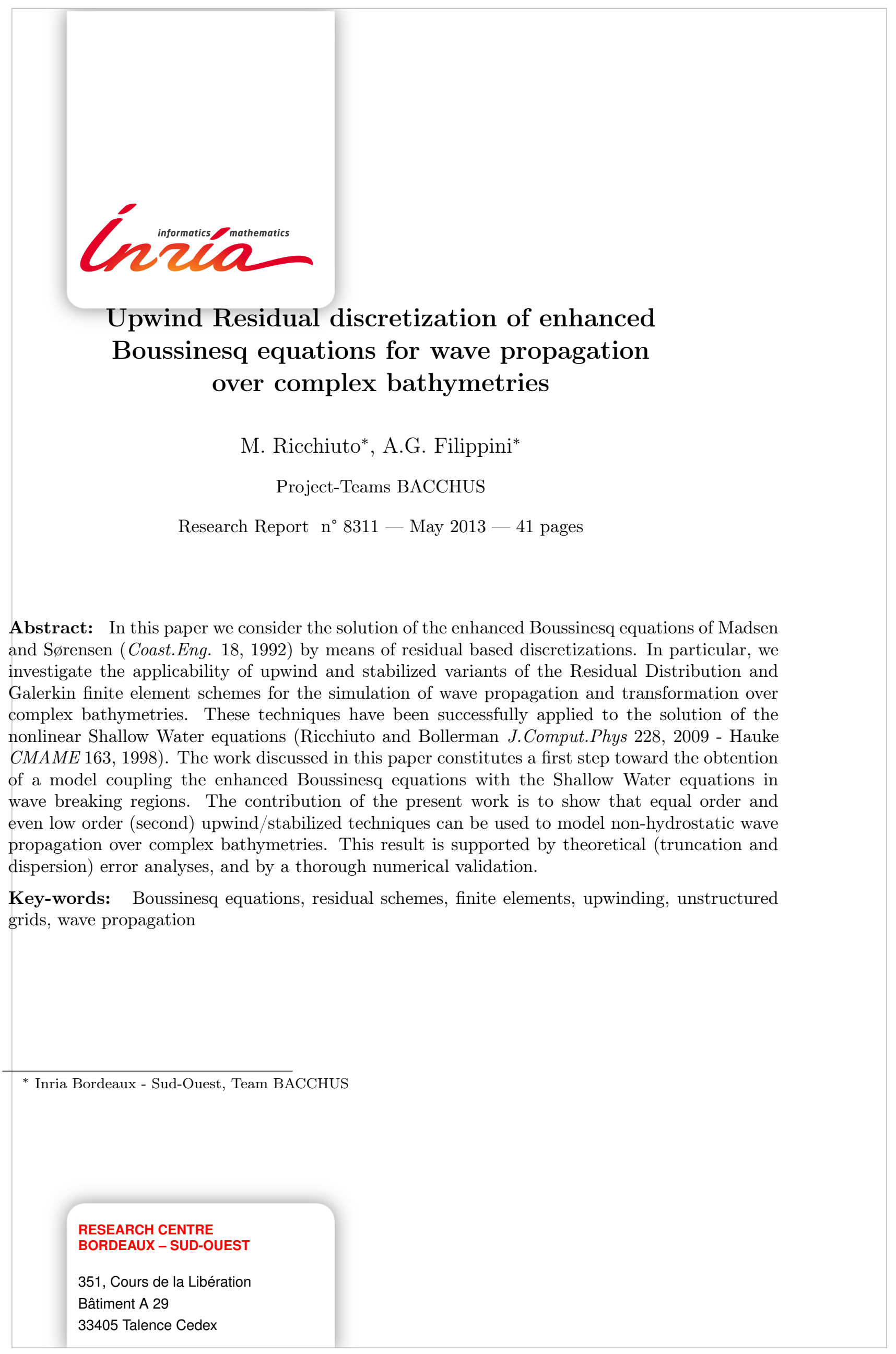




\section{Upwind Residual discretization of enhanced Boussinesq equations for wave propagation over complex bathymetries}

Résumé : Dans ce travail on considère la resolution des equations de Boussinesq proposées par Madsen et Sørensen (Coast.Eng. 18, 1992) avec de mthodes "residual based". On étudie en particulier la possibilité d'utiliser des formulation décentrées de méthodes de type "continuous Galerkin" et "Residual Distribution" pour la simulation de la propagation et transformation de vagues sur bathymetries complexes. Ces méthodologies on étés utilisées avec succès pour la résolution des équations Shallow Water (Ricchiuto and Bollerman J.Comput.Phys 228, 2009 - Hauke CMAME 163, 1998). Ce travail constitue un premier pas dans l'obtention d'un modèle complet qui couple les équations de Boussinesq pour la propagation des vagues avec les équations Shallow Water dans la région de déferlement. La contribution de cet article est de montrer que meme avec des interpolation surface libre/vitesse d'ordre deux des techniques type éléments finis upwind peuvent etre utilisées pour des modèles non-hydrostatiques de type Boussinesq. Ce résultat est confirmé par des analyses d'erreur et par des nombreux études numériques.

Mots-clés : Modèles de Boussinesq, schémas residual based, éléments finis, upwinding, maillages non-structurés, propagation de vagues 


\section{Contents}

$\begin{array}{lll}1 & \text { Introduction } & 3\end{array}$

$\begin{array}{|ll|}2 & \text { Enhanced Boussinesq equations in one dimension }\end{array}$

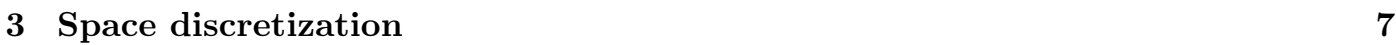

3.1 Generalities and notation . . . . . . . . . . . . . . . . . 7

3.2 Continuous Galerkin approximation $\ldots \ldots \ldots$. . . . . . . . . . . . . . . . . . . . . . .

3.3 Centered Residual Distribution . . . . . . . . . . . . . . . . . . . . . 8

3.4 Upwind discretizations . . . . . . . . . . . . . . . . . . . . . . . . . . 10

3.5 Treatment of the auxiliary variables . . . . . . . . . . . . . . . . . 11

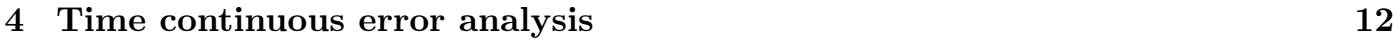

4.1 Truncation error $\ldots \ldots \ldots \ldots \ldots$

4.2 Dispersion error.$\ldots \ldots \ldots \ldots \ldots$

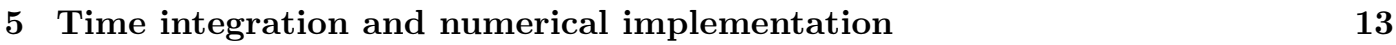

6 Boundary and initial conditions $\quad 15$

6.1 Boundary conditions . . . . . . . . . . . . . . . . . . . 15

6.2 Solitary wave generation . . . . . . . . . . . . . . . . . . . . . . . . . . . . . . . . . . . . . . . . .

6.3 Internal wave generation $\ldots \ldots \ldots \ldots \ldots$. . . . . . . . . . . . . . . . . . . . . . . . .

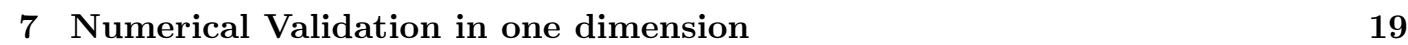

7.1 Solitary wave propagation : grid convergence . . . . . . . . . . . . . . . 19

7.2 Head-on collision of two solitary waves . . . . . . . . . . . . . . . . . . . . . . 19

7.3 Wave propagation over a shelf . . . . . . . . . . . . . . . . . . . . . . . . 20

7.4 Periodic wave propagation over a submerged bar . . . . . . . . . . . . . . . . 21

8 Preliminary validation in two space dimensions $\quad 23$

8.1 Enhanced Boussinesq equations in two space dimensions . . . . . . . . . . . . . 23

8.2 SUPG scheme : implementation in two dimensions . . . . . . . . . . . . . . 24

8.3 Wave diffraction over a semi-circular shoal . . . . . . . . . . . . . . . . . . . 27

8.4 Wave diffraction over an elliptic shoal . . . . . . . . . . . . . . . . . . . . . . 29

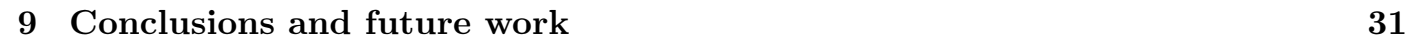

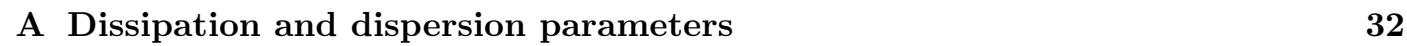

A.1 Second order central finite differences . . . . . . . . . . . . . . . . . . . . . . . . . . . . . .

A.2 Fourth order central finite differences . . . . . . . . . . . . . . . . . . . . . . . . . . . . . . . . . . .

A.3 Continuous Galerkin scheme . . . . . . . . . . . . . . . . . . . . . . . 33

A.4 Centered residual distribution . . . . . . . . . . . . . . . . . . . . 34

A.5 SUPG scheme . . . . . . . . . . . . . . . . . . . . . . 35

A.6 Upwind residual distribution . . . . . . . . . . . . . . . . . . . 36

\section{Introduction}

The accurate simulation of nonlinear and non-hydrostatic wave propagation and transformation on complex bathymetries in the near shore region, up to the shoreline, plays a major role in coastal engineering. Numerical models for the applications involved benefit on one hand from the development of mathematical models with improved dispersion and shoaling characteristics, and, on the other, from the availability of accurate and stable discretizations of these equations. 
Significant effort has been put in the last 20 years in development of systems of depth averaged equations which correctly reproduce the dispersion characteristics of wave propagation in the near shore region. Starting from the Boussinesq equations of Peregrine [42], several improved and enhanced Boussinesq models have been proposed over the years, including, among those having the largest impact in literature and the most recent ones, the enhanced equations of Madsen and Sørensen [41, the extended formulation of Nwogu [39], genuinely nonlinear Serre-Green-Naghdi equations [36], and nonlinear and non-hydrostatic higher order Shallow-Water type models [27]. These models have been obtained by retaining asymptotic behavior of the order of $\mathscr{O}\left(\mu^{2}\right), \mu$ being the ratio of water depth to wavelength. If $h_{0}$ is the value of the depth at sill water, they give a correct description of the physics for values of the wave parameter $k h_{0} \approx 3-5$. More accurate models, including effects up to the $\mathscr{O}\left(\mu^{4}\right)$ order have been proposed e.g. in [26].

Concerning the numerics used to solve these equations, the literature is full of promising models involving finite differences, finite volumes, and finite elements approaches. The major challenges that need to be dealt with are the approximation of the complex higher order derivative terms present in all non-hydrostatic depth-averaged models, the accuracy requirements on the schemes in terms of low dispersion error. In addition, Boussinesq models can be coupled with the nonlinear Shallow-Water (NLSW) equations to model wave breaking [13, 49. This requires the underlying numerics used for the Boussinesq equations to robustly handle the Shallow water limit.

The presence of higher order (third) partial derivatives has made the use of finite difference approximations appealing and quite popular (see e.g. [10, 24, 39, 25] to cite a few). The main drawback of the finite difference approach is the need of structured spatial meshes, even for irregular domains, and poor local mesh adaptivity potential (even tough hierarchical block structured multi-level approaches do exist, see e.g. [11]).

Fully unstructured solvers allowing for adaptive mesh refinement have been proposed, based either on the finite volume, or on the finite element approach. To the author's knowledge, genuinely multidimensional unstructured finite volume discretizations of enhanced Boussinesq equations have been actually proposed only in [35, 8], other works proposing some form of hybridization of finite volume/finite difference schemes on structured meshes or even in one space dimension (see e.g. [20, 14, 50] and references therein). The results presented in 35] are particularly encouraging, and the extension of the authors' model to wave breaking applications, presented at the Modeling the Earth system conference in Boulder [34, has shown the high potential of their approach. One criticism that can be made to the finite volume framework is that going beyond third order of accuracy might be quite hard, due to the necessity of introducing higher order multidimensional reconstructions for both the unknowns, and for the velocity divergence, to allow the discretization of the dispersive terms [35. The advantage of the finite volume framework is of course a very robust approximation of the NLSW limit.

On the other hand, the finite element approximation gives a framework to naturally introduce higher order polynomial representation of the unknowns and of their derivatives, simply by handling these as auxiliary variables. The work of [23, 22] on discontinuous Galerkin approximations of enhanced Boussinesq models shows the potential in terms of accuracy of the finite element approach. Continuous Galerkin discretizations of Bossinesq models have been proposed by several authors. For example, in [17] a Taylor-Galerkin formulation for the Peregrine equations is duscussed. More recently, a model based on Taylor-Galerkin time integration and the enhanced Madsen and Sørensen equations has been proposed in [40, using mixed approximation space. Standard Galerkin approximations are also discussed in [37, 52] (see also the $\mathrm{PhD}$ [51]). These contributions show results at leas as good as those obtained by means of finite difference schemes, with the additional flexibility of a truly unstructured formulation. 
In this paper we want to add to this panorama an additional element, by analyzing and testing residual based and finite element type schemes which include some form of upwind stabilization and which, in the Shallow Water limit, reduce to well known discretizations of the NLSW equations, namely the Residual Distribution (RD) schemes [46, 45, 18, 15, and upwind stabilized Galerkin schemes (SUPG) 28, 29, 32. These schemes have shown very high potential in handling the NLSW, both in terms of preservation of physically relevant steady equilibria (well-balancedness), and in terms stale approximation of moving shorelines [46, 44, 28, 15. For purely hyperbolic problems, it is known that the presence of a mass matrix leads to improved dispersion characteristics for these schemes, compared to finite difference discretizations. While in the hyperbolic case this might seem like a drawback, in presence of mixed space and time derivatives, as in Boussinesq models, this gives an advantage, allowing to build discretizations that, on a reduced stencil, and even for low order interpolation (piecewise linear), yield dispersion properties similar to those of higher order finite difference schemes. Our aim is to analyze both theoretically and numerically second order upwind RD and SUPG discretizations for the enhanced Boussinesq equations of 41 to asses their applicability to wave propagation. This work has to be understood as a first step toward the construction of a model including coupling with the NLSW equations to handle wave breaking and moving shorelines.

The structure of the paper is the following. In section $\S 2$ we recall the basic form of the enhanced Boussinesq model of [41, and in section $\S 3$ we present the schemes analyzed in the paper. Section $\S 4$ is devoted to a time continuous error analysis and comparison with second and fourth order finite difference discretizations of the linearized equations. Sections $\S 5$ and $\S 6$ discuss details relative to the numerical implementation of the schemes and their validation on one dimensional problems with comparison to analytical solutions and experimental data. Preliminary validation of the schemes in two space dimensions is discussed in section $\S 7$. The paper is ended by a summary of the results and an outlook on ongoing work.

\section{Enhanced Boussinesq equations in one dimension}

With reference to the notation of figure 1, the enhanced Boussinesq equations of Madsen and Sørensen [41] can be written as

$$
\left\{\begin{array}{l}
\partial_{t} \eta+\partial_{x} q=0 \\
\partial_{t} q-B h^{2} \partial_{x^{2} t} q-\frac{1}{3} h \partial_{x} h \partial_{x t} q+\partial_{x}(u q)+g H \partial_{x} \eta+ \\
-\beta g h^{3} \partial_{x^{3}} \eta-2 \beta g h^{2} \partial_{x} h \partial_{x^{2}} \eta=0
\end{array}\right.
$$

where $\eta(x, t)$ and $h(x)$ deote the surface elevation and the depth at still water (cf. figure 1), while $H(x, t)=\eta(x, t)+h(x)$ and $q(x, t)$ are the total depth, and the discharge $q=H u, u$ denoting the depth averaged speed, as in the NSWE system. In addition, the brief notation $\partial_{x^{n}}$ will be used within this work in order to indicate the recursive application of the partial derivative with respect to $x$ for $n$ times. These equations provide a description of $\mathscr{O}\left(\epsilon, \mu^{2}\right)$ of the wave propagation physics, recalling that the nonlinearity $\epsilon$ parameter represents the ratio of wave amplitude to depth, and the dispersion $\mu$ is the ratio of water depth to wavelength.

This model is weakly nonlinear, preserving the same shallow water terms $\partial_{x}(u q)$ and $g H \partial_{x} \eta$ which are the only nonlinear in the system, being, thus, different from the fully nonlinear models, like the one proposed in [36]. It also contains additional dispersive terms in the momentum equation, which are linear with respect to the unknowns $\eta$ and $q$ of the system. These are pre-multiplied by two numerical parameters $B$ and $\beta$, whose values are obtained by optimizing the dispersion properties of the linearized model with respect to the Airy wave theory. In such a way, the two parameters assume the values $\beta=1 / 15$ and $B=\beta+1 / 3$ [41].

For later use, we recall that, assuming that both $\eta$ and $u$ are very small perturbation of 


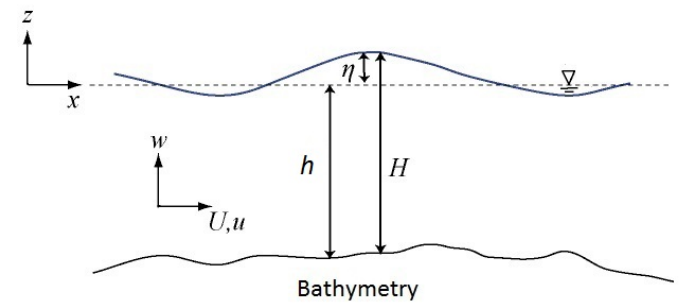

Figure 1: Sketch of the free surface flow problem, main parameters description.

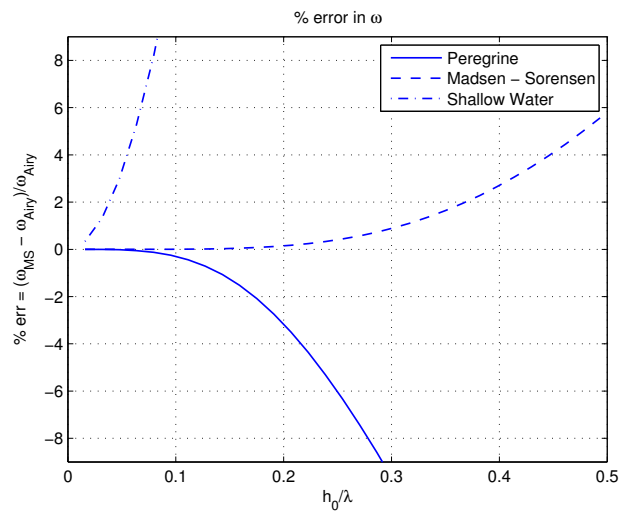

Figure 2: Phase error w.r.t. the Airy theory.

a still steady state, and flat bathymetry, the linearized version of system i1 reads

$$
\left\{\begin{array}{l}
\partial_{t} \eta+h_{0} \partial_{x} u=0 \\
\partial_{t} u-B h_{0}^{2} \partial_{x^{2} t} u+g \partial_{x} \eta-\beta g h_{0}^{2} \partial_{x^{3}} \eta=0
\end{array}\right.
$$

The linearized Boussinesq equations of Peregrine are obtained by setting $\beta=0$, while the NLSW equations are recovered by neglecting all the high order derivative terms. The dispersion characteristics of this linearized system are obtained by replacing $\eta$ and $u$ by a propagating Fourier mode

$$
W=\left(\begin{array}{l}
\eta \\
u
\end{array}\right)=W_{0} e^{\nu t+\mathrm{i} k x}
$$

with i the imaginary unit, and with the wavenumber $k$ related to the wavelength as $k=2 \pi / \lambda$, and with a complex amplification parameter $\nu=\xi+\mathrm{i} \omega, \xi$ representing the dissipation parameter, and $\omega$ the phase. The so-called Airy theory for water wave propagation (see $e . g$. [39] and references therein) gives for these parameters the analytical values

$$
\left\{\begin{array}{l}
\xi_{\text {Airy }}=0 \\
\omega_{\text {Airy }}^{2}=C_{0}^{2} k^{2} \frac{\tanh \left(k h_{0}\right)}{k h_{0}}
\end{array}\right.
$$

with $C_{0}^{2}=g h_{0}$, the linearized Shallow Water celerity. Substitution of the Fourier mode in (2) quickly provides the approximated value given by the Madsen and Sørensen model

$$
\left\{\begin{array}{l}
\xi_{\mathrm{MS}}=0 \\
\omega_{\mathrm{MS}}^{2}=C_{0}^{2} k^{2} \frac{1+\beta\left(k h_{0}\right)^{2}}{1+B\left(k h_{0}\right)^{2}}
\end{array}\right.
$$


These expressions are compared with the analytical one on figure 2 , showing the improvement of the present model w.r.t. both Peregrine's equations and the NLSW system.

\section{Space discretization}

\subsection{Generalities and notation}

Let $\Omega$ denote the spatial domain. We consider a tesselation $\Omega_{\mathrm{h}}$ composed by a set of nonoverlapping elements, the subscript $\mathrm{h}$ denoting the reference mesh size. The generic element $\mathrm{K}$ is defined by a set of nodes, e.g. in one space dimension $\mathrm{K} \equiv\left[x_{j}, x_{j+1}\right]$, with $\mathrm{h}_{K}=x_{j+1}-x_{j}$. Unknowns are stored at nodes as time dependent values $\left\{\eta_{i}(t)\right\}_{i \geq 1}$ and $\left\{q_{i}(t)\right\}_{i \geq 1}$. For a generic node $i$ we will also denote by $\mathrm{K}_{i}$ the set of elements containing $i$ as a node. As in the standard $P^{1}$ finite element method, nodal values are interpolated by means of piecewise linear continuous shape functions $\varphi_{i}(x)$, the interpolated values being denoted by $\eta_{h}$, and $q_{h}$ with (cf. figure 3)

$$
\begin{aligned}
\eta_{\mathrm{h}}(t, x) & =\sum_{i \geq 1} \eta_{i}(t) \varphi_{i}(x)=\sum_{K} \sum_{j \in K} \eta_{j}(t) \varphi_{j}(x) \\
q_{\mathrm{h}}(t, x) & =\sum_{i \geq 1} q_{i}(t) \varphi_{i}(x)=\sum_{K} \sum_{j \in K} q_{j}(t) \varphi_{j}(x)
\end{aligned}
$$

with the $\varphi_{i}(x)$ the standard continuous piecewise linear finite element basis functions assuming value 1 in node $i$ and zero in all the other nodes. As discussed in the introduction, in this paper we focus on piecewise linear interpolation in order to show the feasibility of the use of compact low order discretizations for wave propagation. However all the developments presented, including the general form of the schemes, extend naturally to higher order polynomial approximation.
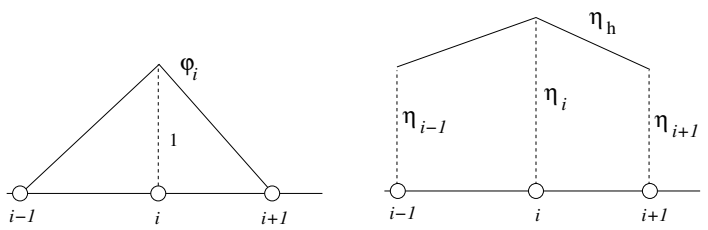

Figure 3: $P^{1}$ finite element interpolation

In the following subsections we present the schemes studied in the paper in the hypothesis that periodic boundary conditions are used. More details concerning boundary conditions and wave generation are given in sections $\S 5$ and $\S 6$. 


\subsection{Continuous Galerkin approximation}

Following [52, we write the continuous Galerkin approximation of system (1) as, seek $\forall, i \in$ $\Omega_{h}$, the solution of

$$
\begin{aligned}
& \int_{\Omega_{\mathrm{h}}} \varphi_{i} \partial_{t} \eta_{\mathrm{h}}-\int_{\Omega_{\mathrm{h}}} q_{\mathrm{h}} \partial_{x} \varphi_{i}=0 \\
& \int_{\Omega_{\mathrm{h}}} \varphi_{i} \partial_{t} q_{\mathrm{h}}+\int_{\Omega_{\mathrm{h}}} B \partial_{x t} q_{\mathrm{h}} \partial_{x}\left(h^{2} \varphi_{i}\right)-\int_{\Omega_{\mathrm{h}}} \frac{1}{3} \varphi_{i} h \partial_{x} h \partial_{x t} q_{\mathrm{h}}-\int_{\Omega_{\mathrm{h}}}(u q)_{\mathrm{h}} \partial_{x} \varphi_{i}-\int_{\Omega_{\mathrm{h}}} g \frac{H_{\mathrm{h}}^{2}}{2} \partial_{x} \varphi_{i}-\int_{\Omega_{\mathrm{h}}} \varphi_{i} g H_{\mathrm{h}} \partial_{x} h \\
& \quad-\int_{\Omega_{\mathrm{h}}} \varphi_{i} \beta g h^{3} \partial_{x} w_{\mathrm{h}}^{\eta}-\int_{\Omega_{\mathrm{h}}} \varphi_{i} 2 \beta g h^{2} \partial_{x} h w_{\mathrm{h}}^{\eta}=0 \\
& \int_{\Omega_{\mathrm{h}}} \varphi_{i} w_{\mathrm{h}}^{\eta}+\int_{\Omega_{\mathrm{h}}} \partial_{x} \eta_{\mathrm{h}} \partial_{x} \varphi_{i}=0
\end{aligned}
$$

where the auxiliary variable $w^{\eta}$ is an approximation to the second order spatial derivative of the free surface level $\eta$. Note that in (6) we have separated and integrated by parts the terms corresponding to the NLSW flux terms $\partial_{x}(u q)$ and $g \partial_{x} H^{2} / 2$. However, due to the piecewise continuous nature of the approximation, and to the assumed periodic boundary conditions, the terms corresponding to these fluxes can be re-integrated by parts giving

$$
-\int_{\Omega_{\mathrm{h}}}(u q)_{\mathrm{h}} \partial_{x} \varphi_{i}=\sum_{\mathrm{K} \in \Omega_{\mathrm{h}}} \int_{\mathrm{K}} \varphi_{i} \partial_{x}(u q)_{\mathrm{h}}-\sum_{\mathrm{K} \in \Omega_{\mathrm{h}}}\left[\varphi_{i}(u q)_{\mathrm{h}}\right]_{\mathrm{K}}=\sum_{\mathrm{K} \in \Omega_{\mathrm{h}}} \int_{\mathrm{K}} \varphi_{i} \partial_{x}(u q)_{\mathrm{h}}=\int_{\Omega_{\mathrm{h}}} \varphi_{i} \partial_{x}(u q)_{\mathrm{h}}
$$

the sum of jump terms on element boundaries $\left[\varphi_{i}(u q)_{\mathrm{h}}\right]_{\mathrm{K}}$ being zero due to the continuity of $\varphi_{i}$ and $(u q)_{\mathrm{h}}$, and to the compact support of the basis functions. Proceeding similarly for the $g \partial_{x} H^{2} / 2$ term, we can recast $(6)$ as

$$
\begin{aligned}
& \int_{\Omega_{\mathrm{h}}} \varphi_{i} \partial_{t} \eta_{\mathrm{h}}-\int_{\Omega_{\mathrm{h}}} q_{\mathrm{h}} \partial_{x} \varphi_{i}=0 \\
& \int_{\Omega_{\mathrm{h}}} \varphi_{i} \partial_{t} q_{\mathrm{h}}+\int_{\Omega_{\mathrm{h}}} B \partial_{x t} q_{\mathrm{h}} \partial_{x}\left(h^{2} \varphi_{i}\right) \\
& \quad+\int_{\Omega_{\mathrm{h}}} \varphi_{i}\left[-\frac{1}{3} h \partial_{x} h \partial_{x t} q_{\mathrm{h}}+\partial_{x}(u q)_{\mathrm{h}}+g H_{\mathrm{h}} \partial_{x} \eta_{\mathrm{h}}-\beta g h^{3} \partial_{x} w_{\mathrm{h}}^{\eta}-2 \beta g h^{2} \partial_{x} h w_{\mathrm{h}}^{\eta}\right]=0 \\
& \int_{\Omega_{\mathrm{h}}} \varphi_{i} w_{\mathrm{h}}^{\eta}+\int_{\Omega_{\mathrm{h}}} \partial_{x} \eta_{\mathrm{h}} \partial_{x} \varphi_{i}=0
\end{aligned}
$$

The actual discretization is obtained by evaluating all the integrals by numerical quadrature over each element $\mathrm{K} \in \mathrm{K}_{i}$, with the assumption of piecewise linear variation of all the quantities involved. Note that the introduction of the auxiliary variable $w^{\eta}$ is made necessary by the presence of the higher (third) order derivatives of the free surface level.

\subsection{Centered Residual Distribution}

Even though sharing a similar cell-vertex philosophy, the Residual Distribution (RD) discretization is obtained with a different strategy. Discrete equations are obtained by first 
computing integrated values of the elemental residuals, denoted by $\Phi^{K}$, and then by distributing fractions of these elemental residuals to the nodes forming the element (cf. figure 4 and refer to [18, 46, 45] for details).
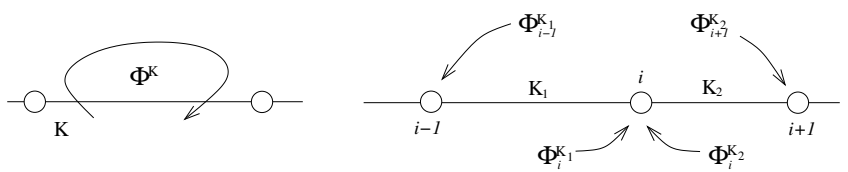

Figure 4: One dimensional Residual Distribution

In particular, with the same underlying continuous spatial approximation used for the Galerkin scheme, we define the element residual $\Phi^{K}=\left[\begin{array}{ll}\Phi_{\eta}^{\mathrm{K}} & \Phi_{q}^{\mathrm{K}}\end{array}\right]^{T}$ as

$$
\begin{aligned}
\Phi_{\eta}^{\mathrm{K}}=\int_{\mathrm{K}}\left(\partial_{t} \eta_{\mathrm{h}}\right. & \left.+\partial_{x} q_{\mathrm{h}}\right) \\
\Phi_{q}^{\mathrm{K}}=\int_{\mathrm{K}}\left(\partial_{t} q_{\mathrm{h}}\right. & -B h^{2} \partial_{x^{2} t} q_{\mathrm{h}}-\frac{1}{3} h \partial_{x} h \partial_{x t} q_{\mathrm{h}}+\partial_{x}(u q)_{\mathrm{h}} \\
& \left.\quad+g H_{\mathrm{h}} \partial_{x} \eta_{\mathrm{h}}-\beta g h^{3} \partial_{x^{3}} \eta_{\mathrm{h}}-2 \beta g h^{2} \partial_{x} h \partial_{x^{2}} \eta_{\mathrm{h}}\right)
\end{aligned}
$$

Discrete equations for the nodal values are obtained by numerically integrating the relations defining the elemental residual (8), and then by distributing the resulting quantity to the nodes of element $\mathrm{K}$ by means of a distribution matrix $\beta_{i}^{\mathrm{K}}$, so that nodal discrete equations are obtained as

$$
\sum_{\mathrm{K} \in \mathrm{K}_{i}} \beta_{i}^{\mathrm{K}} \Phi^{\mathrm{K}}=0
$$

The centered RD scheme (cRD) is obtained (in one space dimension) with the simple choice $\beta_{i}^{\mathrm{K}}=\mathrm{I}_{2} / 2 \forall i$ and $\forall \mathrm{K}$, with $\mathrm{I}_{2}$ denoting the $2 \times 2$ identity matrix.

As in the case of the Galerkin scheme, the presence of high order derivative terms requires the definition of nodal values of the derivatives of the variables, which are otherwise only defined at the elemental level. As in finite element schemes, this is achieved in the RD community by treating these nodal derivatives as a set of auxiliary variables, for which reconstruction strategies or ad-hoc discrete equations are developed (see e.g. 2, 3, 43, 38, and references therein). Following the work done in [2, 3, 43, and using the auxiliary variable already introduced for the Galerkin scheme (cf. equation (7)), we have in practice computed the second in $(8)$ as

$$
\begin{aligned}
\Phi_{q}^{\mathrm{K}}=\int_{\mathrm{K}}\left(\partial_{t} q_{\mathrm{h}}\right. & -B h^{2} \partial_{x t} w_{\mathrm{h}}^{q}-\frac{1}{3} h \partial_{x} h \partial_{x t} q_{\mathrm{h}}+\partial_{x}(u q)_{\mathrm{h}} \\
& \left.+g H_{\mathrm{h}} \partial_{x} \eta_{\mathrm{h}}-\beta g h^{3} \partial_{x} w_{\mathrm{h}}^{\eta}-2 \beta g h^{2} \partial_{x} h w_{\mathrm{h}}^{\eta}\right)
\end{aligned}
$$

with auxiliary variables given by

$$
\begin{gathered}
\int_{\Omega_{\mathrm{h}}} \varphi_{i} w_{\mathrm{h}}^{q}+\int_{\Omega_{\mathrm{h}}} q_{\mathrm{h}} \partial_{x} \varphi_{i}=0 \\
\int_{\Omega_{\mathrm{h}}} \varphi_{i} w_{\mathrm{h}}^{\eta}+\int_{\Omega_{\mathrm{h}}} \partial_{x} \eta_{\mathrm{h}} \partial_{x} \varphi_{i}=0
\end{gathered}
$$

where now the auxiliary variable $w^{q}$ is an approximation of the nodal derivative of $q$. More details on the treatment of these variables will be given in section $§ 3.5$. 


\subsection{Upwind discretizations}

Schemes (7) and (8)-(10) are centered approximations of the equations and are not well suited for the discretization of the Shallow Water limit for which some form of upwinding is necessary to stabilize the system, or to provide positivity corrections in correspondence of moving shorelines and discontinuities [45, 46]. In view of the coupling of the Boussinesq equations with the NLSW system to handle wave breaking, we will analyze here two upwind schemes obtained by adding to schemes (7) and(8)-(10) an upwind bias based on the characteristic decomposition of the $N L S W$ equations.

We start by rewriting scheme (7) with the short-notation

$$
\mathcal{R}_{i}^{\mathrm{cG}}\left(\eta_{\mathrm{h}}, q_{\mathrm{h}}\right)=0
$$

with $\mathcal{R}_{i}^{\mathrm{cG}}(\eta, q)$ the array whose components are the left hand sides of the first two equations in (7). In order to construct an upwinding operator, we consider now the quasi-linear form of the NLSW equations which can be recast as

$$
\partial_{t}\left(\begin{array}{c}
\eta \\
q
\end{array}\right)+A \partial_{x}\left(\begin{array}{c}
\eta \\
q
\end{array}\right)=0, \quad A=\left(\begin{array}{cc}
0 & 1 \\
c^{2}-u^{2} & 2 u
\end{array}\right)
$$

where $c^{2}=g H$ is the Shallow Water celerity. We recall that matrix $A$ admits a full set of real linearly independent eigenvectors, associated to the two eigenvalues $u \pm c$. Following the SUPG stabilization technique [29, 32, 46, 1, we define the stabilized variant of the continuous Galerkin (7) as

$$
\mathcal{R}_{i}^{\mathrm{cG}}\left(\eta_{\mathrm{h}}, q_{\mathrm{h}}\right)+\sum_{\mathrm{K} \in \Omega_{h}} \int_{\mathrm{K}} A \partial_{x} \varphi_{i} \tau_{\mathrm{K}} r_{\mathrm{h}}^{\mathrm{MS}}=0
$$

where the matrix $\tau_{\mathrm{K}}$ is the so-called SUPG stabilization parameter, and having denoted by $r_{\mathrm{h}}^{\mathrm{MS}}$ the local residual value of the Madsen and Sørensen equations :

$r_{\mathrm{h}}^{\mathrm{MS}}=\left(\begin{array}{c}\partial_{t} \eta_{\mathrm{h}}+\partial_{x} q_{\mathrm{h}} \\ \partial_{t} q_{\mathrm{h}}-B h^{2} \partial_{x^{2} t} q_{\mathrm{h}}-\frac{1}{3} h \partial_{x} h \partial_{x t} q_{\mathrm{h}}+\partial_{x}(u q)_{\mathrm{h}}+g H_{\mathrm{h}} \partial_{x} \eta_{\mathrm{h}}-\beta g h^{3} \partial_{x^{3}} \eta_{\mathrm{h}}-2 \beta g h^{2} \partial_{x} h \partial_{x^{2}} \eta_{\mathrm{h}}\end{array}\right)$

As done previously, to evaluate the integral of the SUPG stabilization, we introduce the auxiliary variables $w^{\eta} \approx \partial_{x^{2}} \eta$ and $w^{q} \approx \partial_{x} q$, and rewrite $r_{\mathrm{h}}^{\mathrm{MS}}$ as

$$
r_{\mathrm{h}}^{\mathrm{MS}}=\left(\begin{array}{c}
\partial_{t} \eta_{\mathrm{h}}+\partial_{x} q_{\mathrm{h}} \\
\partial_{t} q_{\mathrm{h}}-B h^{2} \partial_{x t} w_{\mathrm{h}}^{q}-\frac{1}{3} h \partial_{x} h \partial_{x t} q_{\mathrm{h}}+\partial_{x}(u q)_{\mathrm{h}}+g H_{\mathrm{h}} \partial_{x} \eta_{\mathrm{h}}-\beta g h^{3} \partial_{x} w_{\mathrm{h}}^{\eta}-2 \beta g h^{2} \partial_{x} h w_{\mathrm{h}}^{\eta}
\end{array}\right)
$$

where now all the quantities involved have a piecewise linear variation. In addition, for a $P^{1}$ approximation, the basis functions derivative $\partial_{x} \varphi_{i}$ are constant within each element, thus if a one point linearization of the NLSW Jacobian is used in evaluating the stabilization integral, we are left with

$$
\mathcal{R}_{i}^{\mathrm{cG}}\left(\eta_{\mathrm{h}}, q_{\mathrm{h}}\right)+\sum_{\mathrm{K} \in \Omega_{h}} A^{\mathrm{K}} \partial_{x} \varphi_{i}^{\mathrm{K}} \tau_{\mathrm{K}} \int_{\mathrm{K}} r_{\mathrm{h}}^{\mathrm{MS}}=0
$$

with $A^{\mathrm{K}}$ denoting hte local linearization of the NLSW flux Jacobian. Comparing 15 with (14) and (10), we see that, in the $P^{1}$ case, scheme 15) can be recast as

$$
\mathcal{R}_{i}^{\mathrm{cG}}\left(\eta_{\mathrm{h}}, q_{\mathrm{h}}\right)+\sum_{\mathrm{K} \in \Omega_{h}} A^{\mathrm{K}} \partial_{x} \varphi_{i}^{\mathrm{K}} \tau_{\mathrm{K}} \Phi^{\mathrm{K}}=0
$$

At last, we employ here the definition of the SUPG stabilization parameter allowing to recover the upwind discretization of a first order hyperbolic operator (see e.g. [9, 19] and references 
therein), namely

$$
\tau_{\mathrm{K}}=\frac{1}{\sum_{j \in \mathrm{K}}\left|\partial_{x} \varphi_{j}^{K}\right|}\left|A^{\mathrm{K}}\right|^{-1}
$$

with the absolute value $\left|A^{\mathrm{K}}\right|$ computed by means of standard eigenvalue decomposition. In one space dimension this leads to the SUPG scheme

$$
\mathcal{R}_{i}^{\mathrm{cG}}\left(\eta_{\mathrm{h}}, q_{\mathrm{h}}\right)+\sum_{\mathrm{K} \in \Omega_{h}} \operatorname{sign}\left(\partial_{x} \varphi_{i}^{\mathrm{K}}\right) \frac{\operatorname{sign}\left(A^{\mathrm{K}}\right)}{2} \Phi^{\mathrm{K}}=0
$$

where again the $\operatorname{sign}$ matrix $\operatorname{sign}\left(A^{\mathrm{K}}\right)$ is computed by means of standard eigenvalue decomposition. In one space dimension, the stabilization operator for node $i$ only acts in the cells $\mathrm{K}^{i-1 / 2} \equiv\left[x_{i-1}, x_{i}\right]$ and $\mathrm{K}^{i+1 / 2} \equiv\left[x_{i}, x_{i+1}\right]$ providing the additional terms $\pm \operatorname{sign}\left(A^{\mathrm{K}}\right) \Phi^{\mathrm{K}^{i \mp 1 / 2}} / 2$.

In the RD case, a more intuitive procedure is used to project the elemental residual $\Phi^{\mathrm{K}}$ onto the NLSW characteristics, and split each term according to the sign of the corresponding eigenvalue. For a node $i$ this boils down to the following discrete equations :

$$
\frac{\mathrm{I}_{2}+\operatorname{sign}\left(A^{\mathrm{K}^{i-1 / 2}}\right)}{2} \Phi^{\mathrm{K}^{i-1 / 2}}+\frac{\mathrm{I}_{2}-\operatorname{sign}\left(A^{\mathrm{K}^{i+1 / 2}}\right)}{2} \Phi^{\mathrm{K}^{i+1 / 2}}=0
$$

which can be readily recast as

$$
\mathcal{R}_{i}^{\mathrm{cRD}}\left(\eta_{\mathrm{h}}, q_{\mathrm{h}}\right)+\sum_{\mathrm{K} \in \Omega_{h}} \operatorname{sign}\left(\partial_{x} \varphi_{i}^{\mathrm{K}}\right) \frac{\operatorname{sign}\left(A^{\mathrm{K}}\right)}{2} \Phi^{\mathrm{K}}=0
$$

having denoted by $\mathcal{R}_{i}^{\mathrm{cRD}}$ the algebraic component associated to the centered RD scheme $\left(\beta_{i}^{\mathrm{K}}=\mathrm{I}_{2} / 2\right.$ in equation (9) plus (8) and $\left.(10)\right)$.

\subsection{Treatment of the auxiliary variables}

As defined by equations (7) and (11), the $L^{2}$ projections defining the nodal values of the auxiliary variables $w_{\mathrm{h}}^{q}$ and $w_{\mathrm{h}}^{\eta}$ require the solution of a linear system whose matrix is the Galerkin mass matrix

$$
m_{i j}^{\mathrm{cG}}=\int_{\Omega_{\mathrm{h}}} \varphi_{i} \varphi_{j}
$$

This matrix being symmetric, positive definite, and constant, this system can be solved very efficiently, and its LU decomposition can be actually stored, reducing the reconstruction of the nodal values $\left\{w_{i}^{q}\right\}_{i \geq 1}$ and $\left\{w_{i}^{\eta}\right\}_{i \geq 1}$ to a matrix-vector product.

However, as remarked in [51], in practice, it makes no difference whether the left hand side of the projection operator is evaluated exactly, or if a mass lumping procedure is employed, yielding, in one space dimension

$$
\left(x_{i+1}-x_{i-1}\right) w_{i}^{q}=q_{i+1}-q_{i-1}
$$

and

$$
\frac{x_{i+1}-x_{i-1}}{2} w_{i}^{\eta}=\frac{\eta_{i+1}-\eta_{i}}{x_{i+1}-x_{i}}-\frac{\eta_{i}-\eta_{i-1}}{x_{i}-x_{i-1}}
$$

Both formulas reduce to standard second order central differencing on an equally spaced mesh.

In all the following analysis, and in all the numerical results, we have replaced the $L^{2}$ projections in (7) and (11) by the simpler finite difference reconstruction formulae (21) and 22 . 


\section{Time continuous error analysis}

In this paragraph we present a truncation and dispersion error analysis of the schemes introduced in section $\S 3$. While it is natural to consider the linearized system (2) for the dispersion analysis, for simplicity we will consider equations (2) for the truncation error as well. The analysis is time-continuous, meaning that we do not take into account time integration, thus allowing to better underline differences between the space discretizations considered. In particular, we will study six different schemes : the four described in section $\S 3$, plus the second order centered finite difference scheme, denote by FD2, and the fourth order centered finite difference scheme, denoted by FD4.

The expressions obtained when discretizing (2) are quite lenghty and reporting them in the body of the paper might lead to useless excessive length. The full discrete equations are thus reported in appendix A (equations (44), 45, 46, (47), 48), and (49) for the FD2, FD4, cG, cRD, SUPG, and URD schemes respectively). We limit ourselves to a few preliminary observations. The first is that, by comparing (44) and (46), we see that the only difference between the FD2 and cG schemes is the treatment of the first order time derivatives $\partial_{t} \eta$ and $\partial_{t} u$. The FD2 scheme provides a pointwise approximations

$$
\frac{d \eta_{i}}{d t}, \quad \frac{d u_{i}}{d t}
$$

while the cG scheme yielding a coupling of neighboring nodes via the mass matrix :

$$
\frac{1}{6} \frac{d \eta_{i-1}}{d t}+\frac{2}{3} \frac{d \eta_{i}}{d t}+\frac{1}{6} \frac{d \eta_{i+1}}{d t}, \quad \frac{1}{6} \frac{d u_{i-1}}{d t}+\frac{2}{3} \frac{d u_{i}}{d t}+\frac{1}{6} \frac{d u_{i+1}}{d t}
$$

The two schemes are otherwise identical.

The cRD scheme (47) differs from both FD2 and cG not only for the presence of a mass matrix, different from the Galerkin one, but also for the approximation of the second order derivative in the mixed term $\partial_{x^{2} t} u$ which, due to the introduction of the auxiliary variable $w^{u}$ results in a larger stencil approximation of the second derivative :

$$
\partial_{x x}(\cdot) \approx \frac{(\cdot)_{i+2}-2(\cdot)_{i}+(\cdot)_{i-2}}{2 \Delta x^{2}}
$$

Otherwise the three schemes provide the same approximation of the third order term, and have the same overall stencil.

Lastly, looking at equations (48) and 49 , we can easily identify the terms associated to the streamline upwind integral :

$$
\begin{aligned}
& \eta_{i} \text { equation }:-\frac{C_{0}}{2 g}\left\{\frac{\Delta x}{2}\left(\frac{d u_{i+1}}{d t}-\frac{d u_{i-1}}{d t}\right)-\frac{B h_{0}^{2}}{\Delta x}\left(\frac{d u_{i+2}}{d t}-2 \frac{d u_{i+1}}{d t}+2 \frac{d u_{i-1}}{d t}-\frac{d u_{i-2}}{d t}\right)\right. \\
& \left.\underline{-g\left(\eta_{i+1}-2 \eta_{i}+\eta_{i-1}\right)}-\underline{\underline{\frac{\beta g h_{0}^{2}}{\Delta x^{2}}\left(\eta_{i+2}-4 \eta_{i+1}+6 \eta_{i}-4 \eta_{i-1}+\eta_{i-2}\right)}}\right\}
\end{aligned}
$$

$u_{i}$ equation : $-\frac{g}{2 C_{0}}\left\{\frac{\Delta x}{2}\left(\frac{d \eta_{i+1}}{d t}-\frac{d \eta_{i-1}}{d t}\right)+\underline{h_{0}\left(u_{i+1}-2 u_{i}+u_{i-1}\right)}\right\}$

The dissipative nature of these terms is obvious from the underlined terms, representing second order approximation of second order derivatives, and the doubly underlined term which is a of a fourth order dissipation term. All the remaining terms are associated to the residual character of the scheme and are difference terms balancing the dissipation terms such that an approximation of differences of the full equations is retained. 


\subsection{Truncation error}

The derivation of the expressions of the truncation errors of the schemes requires lengthy calculations. As already said this is done for the linearized system (2), in the hypothesis that the grid spacing is uniform. The detailed expressions of these errors are not essential, and are reported in appendix A. The main conclusion achieved when comparing these expressions is summarized in table 1 in which the leading orders of the truncation errors for the $\eta$ and $u$ equations in system (2) are reported.

\begin{tabular}{||c||c|c|c|c|c|c||}
\hline & FD2 & FD4 & cG & cRD & SUPG & URD \\
\hline \hline $\mathrm{TE}_{\eta}$ & $\Delta x^{2}$ & $\Delta x^{4}$ & $\boldsymbol{\Delta} \mathbf{x}^{4}$ & $\Delta x^{2}$ & $\mathbf{\Delta x}^{3}$ & $\Delta x^{2}$ \\
\hline $\mathrm{TE}_{u}$ & $\Delta x^{2}$ & $\Delta x^{4}$ & $\Delta x^{2}$ & $\Delta x^{2}$ & $\Delta x^{2}$ & $\Delta x^{2}$ \\
\hline \hline
\end{tabular}

Table 1: Leading orders in the truncation error on the linearized Madsen and Sørensen system

On the same stencil of the FD2 and RD schemes, both the cG and the SUPG schemes give a formal error on $\eta$ higher than what could be expected : the cG scheme is consistent to fourth order, and the SUPG to third order of accuracy. While of course it must be stressed that this result is only true on equally spaced grids, this is very interesting when considering the approximation of planar waves, as e.g. in tests involving waves generated far from obstacles and variable bathymetry. Of course this is also true for planar solutions in two space dimensions and on structured grids, if the schemes reduce to their one dimensional form.

\subsection{Dispersion error}

To obtain the discrete dispersion relations of the schemes, we replace in the discretizations of (2) reported in appendix A (equations (44), (45), (46), (47), (48), and (49) for the FD2, FD4, cG, cRD, SUPG, and URD schemes respectively) propagating Fourier modes to the nodal values of $\eta$ and $u$ :

$$
W_{j}=\left(\eta_{j}(t), u_{j}(t)\right)^{T}=W_{0} e^{\nu_{\mathrm{h}} t+\mathrm{i} k x_{j}}
$$

with i the imaginary unit and $k$ the wavenumber. Manipulating the resulting equations, the complex amplification factor $\nu_{\mathrm{h}}$ of the schemes can be obtained as a function of complex exponentials of $k \Delta x$ and of the reduced wavenumber $k h_{0}$. The expressions obtained for all the schemes are reported in appendix A. To visualize the phase errors of all the schemes, we report in figure 5 the ratios $\omega_{\mathrm{h}} / \omega_{\text {Airy }}$ obtained when placing 5 grid-points per wavelength. In all the figures we have reported for comparison the ratios $\omega_{\mathrm{FD} 2} / \omega_{\mathrm{Airy}}, \omega_{\mathrm{FD} 4} / \omega_{\text {Airy }}$, and $\omega_{\mathrm{MS}} / \omega_{\text {Airy }}$ of the continuous system (cf. section $\S 2$, equations (3) and (4)). Ideally all these curves should lay on the line $y=1$.

From the figure we can clearly see that, while the $\mathrm{cRD}$ and URD schemes provide phase errors comparable to those of the FD2 scheme, the cG and SUPG schemes provide a phase error at least as good if not better than the FD4 scheme, the SUPG giving a surprisingly good approximation. Surprisingly, the cRD and URD give nearly identical results.

\section{Time integration and numerical implementation}

In all the applications considered in this paper, we have used a Crank-Nicholson (CN) time integrator. In particular, all the schemes discussed have been applied to the semi-discrete 

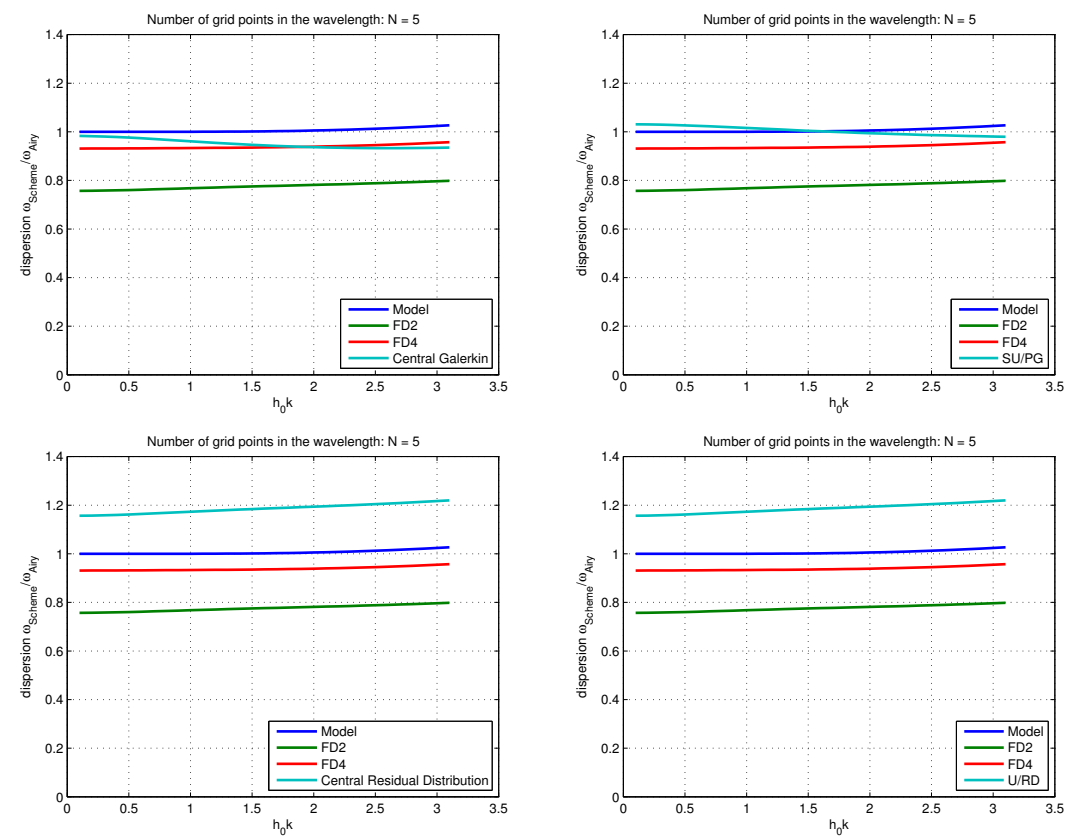

Figure 5: Dispersion characteristics of the schemes with 5 points per wavelength. Top : cG (left) and SUPG (right). Bottom : cRD (left) and URD (right)

version of (1) that reads

$$
\left\{\begin{array}{l}
\Delta^{n+1} \eta+\Delta t \partial_{x} q^{n+1 / 2}=0 \\
\Delta^{n+1} q-B h^{2} \partial_{x^{2}}\left(\Delta^{n+1} q\right)-\frac{1}{3} h \partial_{x} h \partial_{x}\left(\Delta^{n+1} q\right)+\Delta t \partial_{x}(u q)^{n+1 / 2} \\
\quad+\Delta t g H^{n+1 / 2} \partial_{x} \eta^{n+1 / 2}-\Delta t \beta g h^{3} \partial_{x^{3}} \eta^{n+1 / 2}-2 \Delta t \beta g h^{2} \partial_{x} h \partial_{x^{2}} \eta^{n+1 / 2}=0
\end{array}\right.
$$

where $\Delta^{n+1}(\cdot)=(\cdot)^{n+1}-(\cdot)^{n}$, and $(\cdot)^{n+1 / 2}=\left((\cdot)^{n+1}+(\cdot)^{n}\right) / 2$. The choice of the CN integrator is related to its simplicity, its A-stable character, and its non-dissipative nature. Of course, for large time steps the CN scheme will introduce a certain amount of dispersion error. The investigation of the interaction spatial-temporal discretization has to be taken into account, but it has been left out of the present study, and will be the subject of future work.

When applied to (23), all the schemes studied in this paper reduce to a nonlinear algebraic system for the unknowns $\eta_{\mathrm{h}}^{n+1}$ and $q_{\mathrm{h}}^{n+1}$ which can be generally written as

$$
F\left(W_{\mathrm{h}} \mid \eta_{\mathrm{h}}^{n}, q_{\mathrm{h}}^{n}\right)=0
$$

where $W_{\mathrm{h}}$ is the array containing all the nodal values of $\eta_{\mathrm{h}}^{n+1}$ and $q_{\mathrm{h}}^{n+1}$, and with the dependence of the system on the variables at the known time step kept explicitly. System 24) has to be solved by some iterative method. In this work, we have implemented a Newton loop with frozen Jacobian matrix which, can be summarized as follows

1. Set $W_{0}=\left(\eta_{0}^{n+1}, q_{0}^{n+1}\right)^{T}=\left(\eta^{n} q^{n}\right)^{T}$

2. Evaluate the frozen Jacobian matrix

$$
\mathcal{M}=\frac{\partial F}{\partial W_{\mathrm{h}}}\left(W_{\mathrm{h}}=W_{0} \mid \eta_{\mathrm{h}}^{n}, q_{\mathrm{h}}^{n}\right)
$$


3. Compute a $L U$ factorization of $\mathcal{M}$

4. for $\mathrm{k}=1, \mathrm{k}_{\max } \mathrm{do}$

(a) Evaluate $F\left(W_{k-1} \mid \eta_{\mathrm{h}}^{n}, q_{\mathrm{h}}^{n}\right)$

(b) If $\|F\| \leq \epsilon$ set $\mathrm{k}=\mathrm{k}_{\max }$ and exit, else evaluate $W_{k}=W_{k-1}-\mathcal{M}^{-1} F\left(W_{k-1} \mid \eta_{\mathrm{h}}^{n}, q_{\mathrm{h}}^{n}\right)$

5. Set $W_{\mathrm{h}}=W_{\mathrm{k}_{\max }}$

For all the schemes considered so far the evaluation of the Jacobian needed at step 2. is done analytically, while steps 3. and 4.(b) are performed with MUMPS [7, 6], in particular the cost of step 4.(b) is that of 2 matrix-vector products, the bulk of the work being indeed done in steps 2. and 3. The convergence threshold $\epsilon$ is in practice replaced by the $\min \left(\epsilon, \epsilon_{\text {rel }}\left\|F\left(F\left(W_{0} \mid \eta_{\mathrm{h}}^{n}, q_{\mathrm{h}}^{n}\right)\right)\right\|\right.$, with $\epsilon_{\text {rel }}$ a relative tolerance. The typical convergence behavior over a few time steps for all the tests considered in the paper is reported on figure 6 for values of the relative convergece of $10^{-4}$ and $10^{-6}$, and for an absolute convergence threshold of $10^{-12}$. Three sub iterations are in general enough to achieve a relative tolerance of four orders of magnitude, while four or five sub iterations are enough to go in between 6 orders of magnitude w.r.t. the initial residual value and machine accuracy.

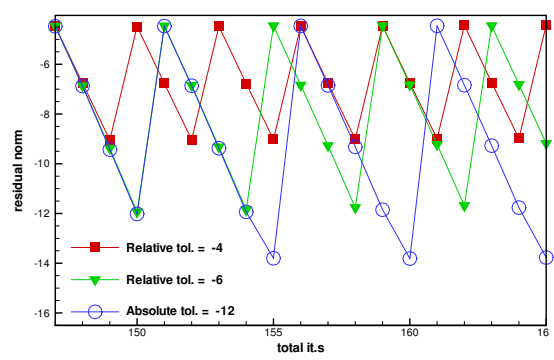

Figure 6: Typical Newton convergence

\section{Boundary and initial conditions}

Before presenting the numerical validation of the schemes, we discuss some important issues related to the solution of (1), namely the techniques used to impose the boundary conditions and, more importantly, the way in which proper initial conditions, including solitary and periodic waves, have been generated.

\subsection{Boundary conditions}

Two types of Boundary Conditions (BC) have been considered here : periodic and outflow conditions. Periodic BCs have been applied quite naturally by adding both the right hand sides and linear system matrix lines (cf. paragraph on Newton solver in section §6) of two coupled periodic nodes.

Outflow BCs represent a bigger challenge since wave reflection at the boundaries might pollute the inner domain solution. As in many other works (see e.g. [51, 52, 21] and references therein), this condition is mimicked here with a viscous sponge layer added to the spatial domain whose sole function is to dump completely all the waves passing through. In these layers, (1) is replaced by

$\mathrm{RR} \mathrm{n}^{\circ} 831$

$$
\left\{\begin{array}{l}
\partial_{t} \eta+\partial_{x} q=\nu \partial_{x^{2}} \eta \\
\partial_{t} q-B h^{2} \partial_{x^{2} t} q-\frac{1}{3} h \partial_{x} h \partial_{x t} q+\partial_{x}(u q)+g H \partial_{x} \eta+ \\
-\beta g h^{3} \partial_{x^{3}} \eta-2 \beta g h^{2} \partial_{x} h \partial_{x^{2}} \eta=\nu \partial_{x^{2}} q
\end{array}\right.
$$


with the additional terms discretized with a Galerkin scheme, independently on the choice of the method used to approximate the rest of the system. Following [51], the viscosity $\nu$ is set to zero everywhere except in the layer $x \in\left[X_{s 1}, X_{s 2}\right]$ where it is defined as:

$$
\nu=n_{1} \frac{e^{n_{2}\left(\frac{x-X_{s 1}}{X_{s 2}-X_{s 1}}\right)}-1}{e-1}
$$

Here $n_{1}$ and $n_{2}$ are constants used to fine tune the amount of viscosity. The values of these coefficients, as well as the length of the layer, are problem dependent. For the problems considered here, we found that for the problems considered here a layer length of approximately $5-10$ meters, and values of the coefficients of $n_{1} \approx 10^{-3}$ and $n_{2} \approx 10$ are a good start, but fine tuning is always preferable to make sure that the amount of reflected information is negligible.

\subsection{Solitary wave generation}

Several test problems involve the propagation of solitary waves, and their interaction with a given bathymetry. An analytical exact soliton for (1) is not available, however, a numerical approximation can be obtained following the derivation of [33]. This derivation and a discussion of the problems encountered in its implementation is reported hereafter.

We seek a solution of (1), on a flat bathymetry with reference depth $h_{0}$, having the self similar behavior

$$
W=(\eta, q)^{T}=W(\zeta)=W(x-C t)
$$

with a certain celerity $C$. We also require this solution and all its derivatives with respect to $\zeta$ to go to zero at infinity. Plugging the expression $W=W(\zeta)$ in (1) with $d=c^{t}=h_{0}$, integrating once between $-\infty$ and $\zeta$, we obtain the relations

$$
\left\{\begin{array}{l}
-C \eta+q=0 \\
-C q+C B h_{0}^{2} q^{\prime \prime}+\frac{q^{2}}{h_{0}+\eta}+g h_{0} \eta+\frac{g}{2} \eta^{2}-\beta g h_{0}^{3} \eta^{\prime \prime}=0
\end{array}\right.
$$

The first relation can be used to derive a second order ODE for $q$, namely :

$$
C h_{0}^{2}\left(B-\beta \frac{C_{0}^{2}}{C^{2}}\right) q^{\prime \prime}+C\left(\frac{C_{0}^{2}}{C^{2}}-1\right) q+\frac{g}{2 C^{2}} q^{2}+C \frac{q}{q+C h_{0}}=0
$$

with $C_{0}^{2}=g h_{0}$. Note that this equation can be pre-multiplied by $q^{\prime}$ and integrated once more between $-\infty$ and $\zeta$ to give

$$
\frac{C h_{0}^{2}}{2}\left(B-\beta \frac{C_{0}^{2}}{C^{2}}\right)\left(q^{\prime}\right)^{2}+\frac{C}{2}\left(\frac{C_{0}^{2}}{C^{2}}-1\right) q^{2}+\frac{g}{6 C^{2}} q^{3}+C q\left(\frac{q}{2}-C h_{0}\right)+C^{3} h_{0}^{2} \ln \frac{q+C h_{0}}{C h_{0}}=0
$$

We have used this equation in two instances. First of all, under the hypothesis that a solitary wave solution does exist, and that in correspondence of its maximum we have $q^{\prime}=0$, and $q=q_{\max }=C A$, with $A$ the amplitude of the wave, we obtain a relation between the wave celerity and the wave amplitude :

$$
\left(\frac{C}{C_{0}}\right)^{2}=\frac{1}{2}\left(\frac{A}{h_{0}}\right)^{2} \frac{1+\frac{1}{3} \frac{A}{h_{0}}}{\frac{A}{h_{0}}-\ln \left(1+\frac{A}{h_{0}}\right)}
$$

Once we set the value of the ratio $A / h_{0}$, we can compute the celerity from $\sqrt{29}$, and integrate (27) as a first order system of ODEs from $\zeta=0$ to $\zeta=\infty$ with initial conditions $q_{0}=C A$, 
and $q_{0}^{\prime}=0$. This has been done numerically in Matlab ${ }^{\circledR}$ using the function ode113. Once the solution for $q$ is obtained, the solitary wave shape is obtained by the first of (26). Unfortunately, the integration of (27) with Matlab ${ }^{\circledR}$ turns out to be quite tricky, and numerical instability appear for very small values of $q$, leading to the behavior illustrated on figure 7 .

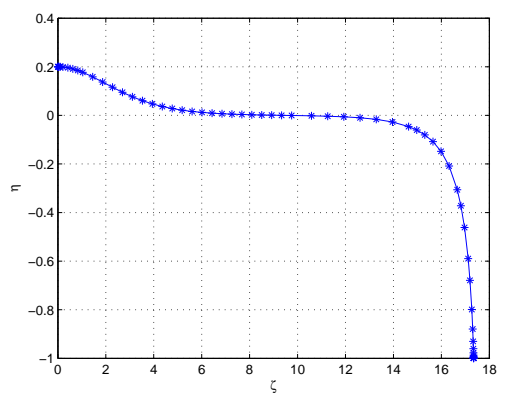

Figure 7: Numerical solution of (27) using the Matlab ${ }^{\circledR}$ solver ode113.

The solution returned by the Matlab ${ }^{\circledR}$ solver reproduces only one half of the soliton shape and is composed by a physical and a non-physical part. Unfortunately, the derivative of the profile obtained never goes to zero, and the acceptable part of the solution stops at values of $\eta / h_{0}$ of $\approx 10^{-4}$ with a large error on the first derivative. In order to cure this and use the profile for a grid convergence analysis, we have tried to improve the resolution of the soliton tail by iteratively solving $(28)$ from the last point in the tail of the numerical soliton minimazing the error between the derivative $q^{\prime}$ obtained numerically by integrating (27), and the analytical one obtained by substituting the numerical value of $q$ into 28 is minimal.
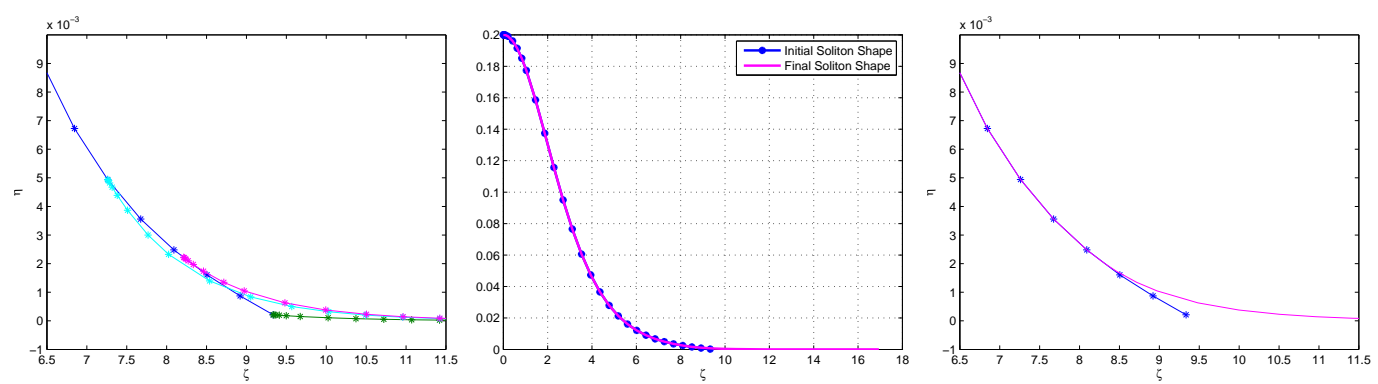

Figure 8: Soliton tail reconstruction by numerical integration of the first order ODE 28)

The result obtained is summarized on figure 8 This procedure has allowed to gain and extra order of magnitude, leading to admissible values of $\eta / h_{0}$ of $\approx 10^{-7}$, reducing the error in the first order derivative. To confirm the validity of the solution, preliminary calculations have been performed with the Galerkin scheme on a very fine mesh. The results for $A / h_{0}=0.2$ are reported in figure 9

\subsection{Internal wave generation}

A large number of tests involve the interaction of monochromatic periodic waves of small amplitude with a given bathymetry. In absence of an exact solution, the generation of such periodic waves is a bit tricky. For very small amplitude waves, some authors suggest the use of Dirichlet type conditions with imposition at the inlet boundary of the condition (see e.g. [50] and references therein)

$$
\left(\eta_{b}(t), q_{b}(t)\right)^{T}=\left(A \sin (\omega t), C_{0} A \sin (\omega t)\right)^{T}
$$

with $C_{0}^{2}=g h_{0}$. We have found numerically the use of this approach relatively inefficient, first due to the fact that the signal actually propagating in the domain had amplitude generally 

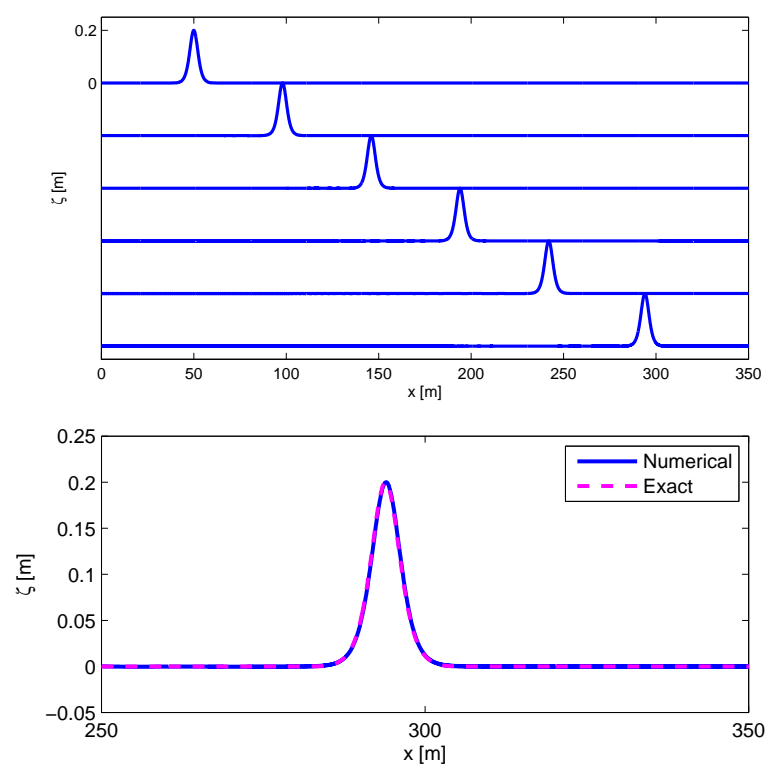

Figure 9: Top : computed evolution of the exact $A / h_{0}=0.2$ soliton. Bottom : comparison between the exact and the numerically computed solitary wave at the final time of the simulation.

larger than $A$, and more importantly due to a very poor iterative convergence in the newton loop, requiring from two to three more iterations to converge.

We have thus chosen a different approach, which is normally quite successfully used of the Boussinesq model of Nwogu [39]. This approach consists in adding to the $\eta$ equation an internal source term of periodic variation in time. Following [51], the first equation of (1) is modified as follows :

$$
\partial_{t}\left(\eta+h_{\mathrm{iwg}}\right)+\partial_{x} q=0
$$

where the form of the internal wave generation term is taken to be:

$$
h_{\text {iwg }}(x, t)=2 C_{0} f_{\text {iwg }}(x) A_{\text {iwg }} \sin (\omega t)
$$

where $C_{0}^{2}=g h_{0}$, and $\omega=2 \pi / T$, with $T$ the period of the required signal. The constant $A_{\text {iwg }}$ controls the amplitude of the signal obtained. Following [51, the spatial dumping function $f_{\text {iwg }}(x)$ is set to

$$
f_{\mathrm{iwg}}(x)=\frac{b_{\mathrm{iwg}}}{\sqrt{\pi}} e^{-b_{\mathrm{iwg}}\left(x-x_{\mathrm{iwg}}\right)^{2}}
$$

These expressions simulate an undulating Gaussian hill centered at the position $x=x_{\text {iwg }}$. The width of the generation region results strongly dependent on the value attributed to the parameter $b_{\text {iwg }}$ which also influences the amplitude of the signal obtained. The values of $A_{\text {iwg }}$ and $b_{\text {iwg }}$ have to be calibrated in order to obtain the signal sought.

Note that, once the first of (1) is recast as (30), the term $h_{\mathrm{iwg}}(x, t)$ is carried along in all the discretization steps together with the $\eta$ term, thus all the time increments $\Delta^{n+1} \eta$ (cf. (23)) are replaced everywhere by $\Delta^{n+1}\left(\eta+h_{\text {iwg }}\right)=\eta^{n+1}+h_{\text {iwg }}^{n+1}-\eta^{n}-h_{\text {iwg }}^{n}$. Typically, the use of this wave generation technique produces a transient signal whose length in time is larger for high frequency and high amplitude waves, and ranges between 10 to 20 seconds. Compared to the approach proposed e.g. in [50], which also involves a transient phase, the main advantage is that Newton convergence remains very fast. Concerning the choice of the parameters in the definition of $h_{\mathrm{iwg}}$, we present an example of a preliminary study in which, 
while of $b_{\text {iwg }}$ is set to $1.5 / T$, with $T$ the period of the signal sought, and the value of $A_{\text {iwg }}$ has been varied from $5 \times 10^{-2}$ to $4 \times 10^{-2}$ for periods of 1 second, 2 seconds, and 4 seconds. The equations are solved with the Galerkin scheme on a relatively fine mesh $(\mathrm{h} \approx 0.005)$. The signal measured 25 meters away from the wave generation source, once the flow has settled to a steady periodic variation, has been analyzed using a Discrete Fourier Transform (DFT). The risults are reportend on figure 10 The pictures show that the signal obtained is basically monochromatic, only very small amplitude high frequencies appearing in the DFT. The relation between the value of $A_{\text {iwg }}$ and the observed amplitude is roughly linear, however fine-tuning is still necessary to be sure that the inlet signal is indeed the one sought.
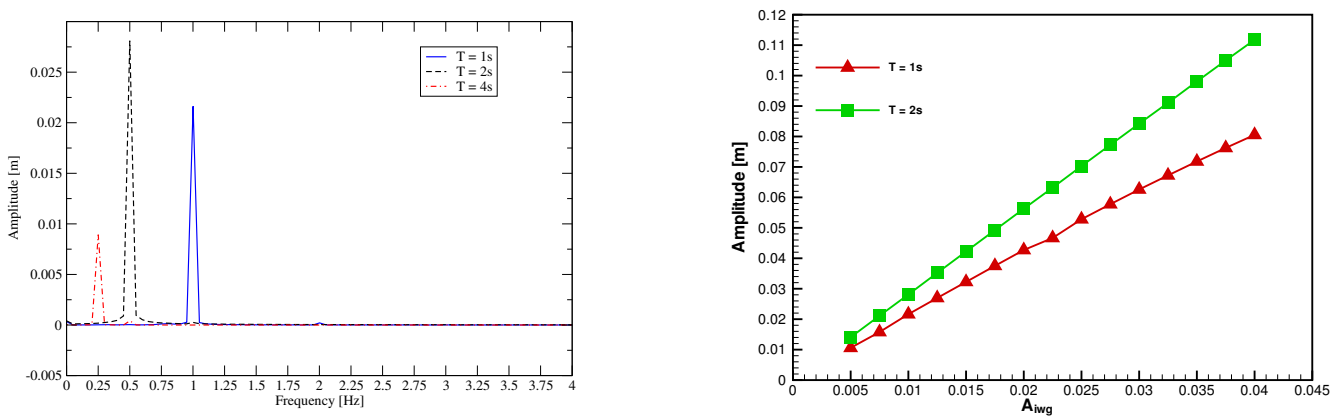

Figure 10: Internal wave generation. Left : DFT of signals obtained for $b_{\text {iwg }}=1.5 / T$ and $A_{\mathrm{iwg}}=0.01$. Right : Amplitude of signals obtained for $b_{\mathrm{iwg}}=1.5 / T$, and $A_{\mathrm{iwg}} \in$ $\left[5 \times 10^{-3}, 4 \times 10^{-2}\right]$.

\section{Numerical Validation in one dimension}

\subsection{Solitary wave propagation : grid convergence}

As a first test, we have verified numerically the accuracy of the schemes studied in the paper by performing a grid convergence study on the solitary wave solutions described in section $\S 6.2$. The solution used for the test is a soliton propagating on a reference depth of $h_{0}=1 \mathrm{~m}$, and with a ratio $A / h_{0}=0.2$. To isolate the error due to the space discretization, the time step has been set for this study to

$$
\Delta t=100 \frac{\Delta x^{3}}{C}
$$

with $C$ the exact celerity of the solitary wave, which, for the parameters chosen here is equal to $C \approx 3.44 \mathrm{~m} / \mathrm{s}$ (a value of $g=9.8066 \mathrm{~m} / \mathrm{s}^{2}$ has been used). The error is computed after the solitary wave has moved of $100 \mathrm{~m}$ from its initial position, corresponding to a final time of $100 / C$.

The results are reported on figure 11 in terms of $L^{2}$ norm of the error in the amplitude $\eta$. The convergence rates obtained confirm the theoretical observations made in section $\S 4$ : the accuracy observed with the RD schemes is lower than that obtained with cG and SUPG which is between 3 and 4 . Surprisingly, for both formulations the effect of the upwind stabilization is barely visible.

\subsection{Head-on collision of two solitary waves}

A common test for the Boussinesq-type and non-hydrostatic models is the simulation of the interaction of two identical solitary waves propagating in opposite directions. After the interactions, one should ideally recover the initial profiles. The collision of the two waves presents additional challenges to the model by a sudden change of the nonlinear and frequency dispersion characteristics. The numerical model must handle the equilibrium between amplitude 


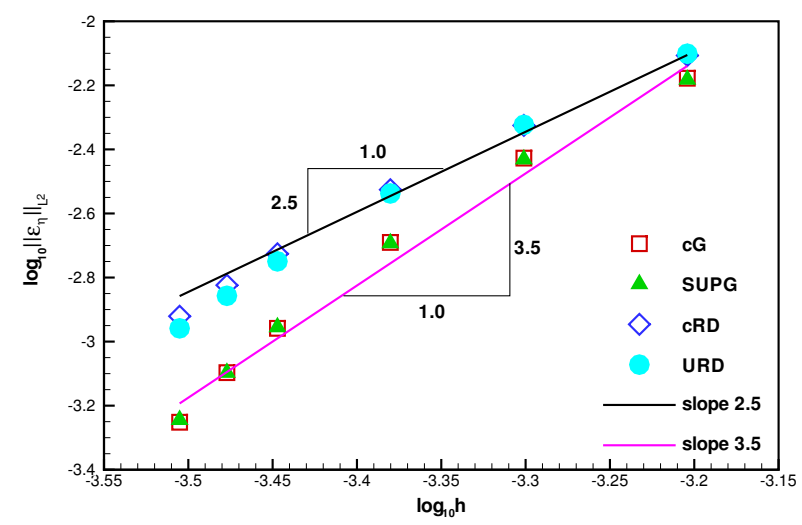

Figure 11: Solitary wave propagation : grid convergence for the cG, cRD, SUPG and URD schemes.

and frequency dispersion to propagate the wave profile at constant shape and speed.

We present here the interaction of two solitons propagating on a depth of $h_{0}=1 \mathrm{~m}$ with amplitude $A / h_{0}=0.2$. The computation uses a grid spacing of $\Delta x=0.08 \mathrm{~m}$ and a time step $\Delta t=0.015 \mathrm{~s}$. The spatial domain is [0 200] $\mathrm{m}$, and the the initial solution is represented in figure 12 .

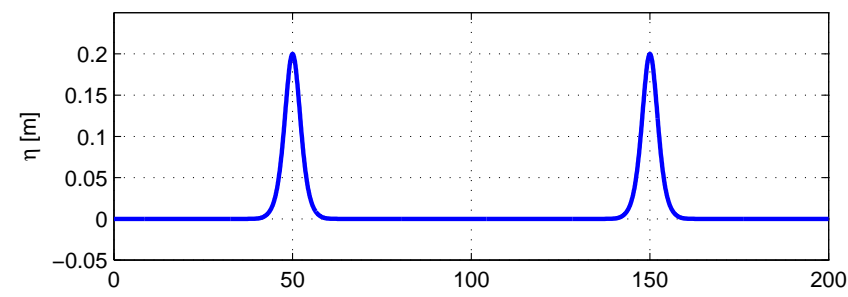

Figure 12: Head on collision of two solitary waves : initial solution.

The results obtained are summarized on figures 13 and 14 . In particular, the pictures on figure 13 show the superposition of the solutions obtained with the four schemes studied in the paper at the time during the interaction when maximum amplitude is reached (left picture) and after the interaction (right picture). No differences can be observed at this scale. On the left on figure 14 the soliton profiles recovered at the final state with the SUPG scheme are plotted against the exact profiles, showing an excellent agreement, while on the right a zoom the the soliton peak on the left is presented. This close up view shows once more the higher resolution of the $\mathrm{cG}$ and SUPG schemes w.r.t. the RD schemes, and also the very similar accuracy of the $\mathrm{cG}$ and SUPG.

\subsection{Wave propagation over a shelf}

We now consider the text case introduced in 33 : a solitary wave of amplitude $A=0.2 \mathrm{~m}$ propagates over a still water level of depth $h_{0}=1 \mathrm{~m}$. At $t=0$ the soliton is placed at $x=80$ $\mathrm{m}$ and it propagates on a shelf where the water depth is reduced to $h=0.5 \mathrm{~m}$ through a ramp of slope of $1: 20$ (figure 15 .

Due to the interaction with the shelf, the solitary splits into several waves. The smallest amplitude of these waves is a reflected on characterized by a very long wavelength, while three forward moving waves are observed after the interaction. The computational domain for this test is [0 280]m. As in [33] we have set the grid spacing to $\Delta x=0.1 \mathrm{~m}$, while the time step has been set to $\Delta t=0.029 \mathrm{~s}$. 

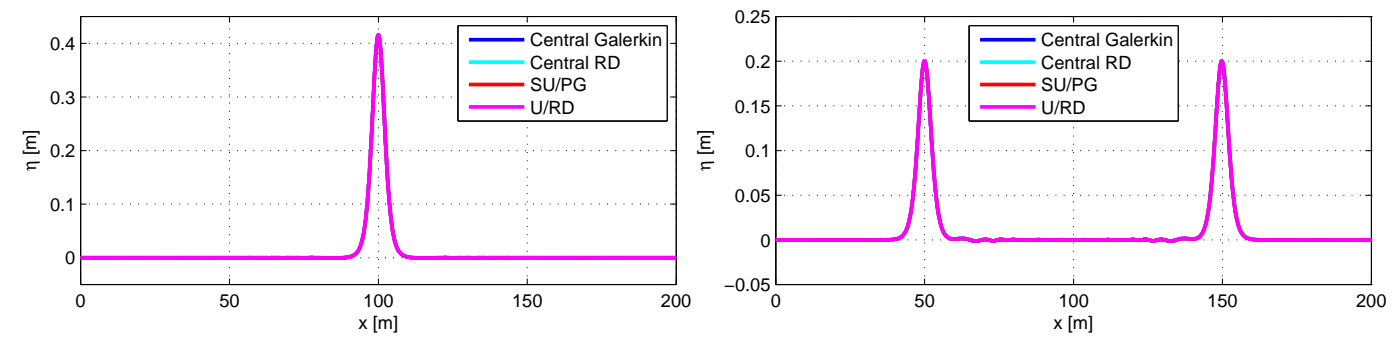

Figure 13: Head on collision of two solitary waves. Left : solution corresponding to maximum amplitude. Right : final state after the interaction. The solutions of all the schemes are superposed.
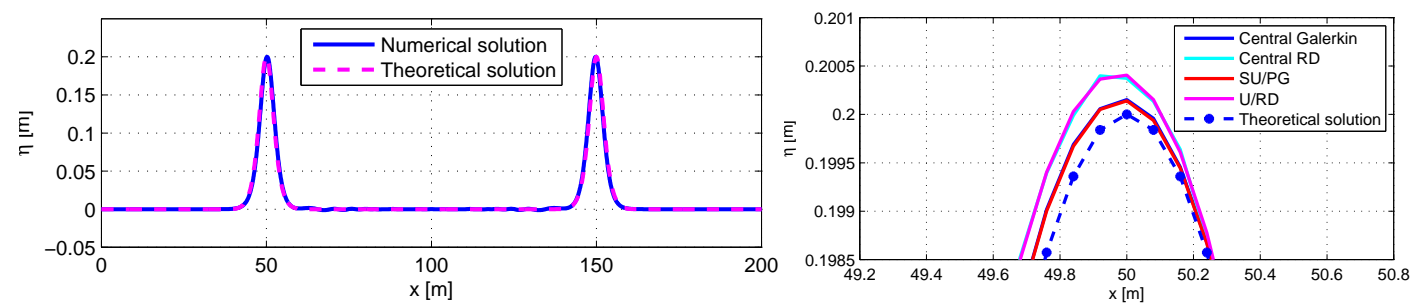

Figure 14: Head on collision of two solitary waves. Left : final state after the interaction, comparison of the SUPG solution with the exact soliton profiles. Right : final state after the interaction, close up view of the schemes resolution of the soliton peak.

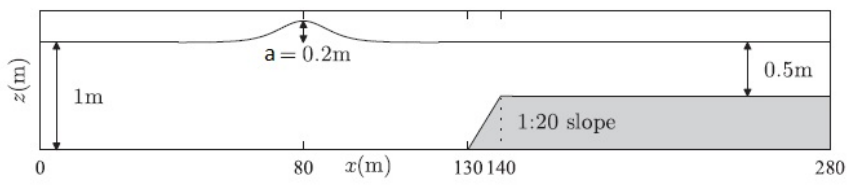

Figure 15: Sketch of the submerged shelf test.

A visualization of the wave transformation in time computed by the SUPG scheme is reported on figure 16, showing the nice capturing of the wave shoaling and splitting due to the interaction with the variable bathymetry. The result compares very well with that reported in 33 .

On figure 17 we compare the the results computed by the different schemes. All of them give an accurate description of the physics of the interaction. A closer look at the highest amplitude wave generated reveals a difference in phase and amplitude between the RD schemes and the cG and SUPG yielding very close results. This is certainly a consequence of the different phase error observed for the schemes (cf. section $\S 4$ ).

\subsection{Periodic wave propagation over a submerged bar}

In 47] a series of laboratory experiments investigating wave propagation and dispersion over a submerged bar are described. The experimental data collected are often used to validate non-hydrostatic models for wave propagation. In particular, we consider here the experiment with the configuration shown in figure 18 . The test consists in a periodic wave of period $T=2.02 \mathrm{~s}$ and amplitude $A=0.01 \mathrm{~m}$ which propagates into an initially undisturbed region of depth $h_{0}=0.4 \mathrm{~m}$ before reaching a bar of the shape and proportions given in the figure.

The numerical set for this test case needs the use of the periodic internal wave generator 


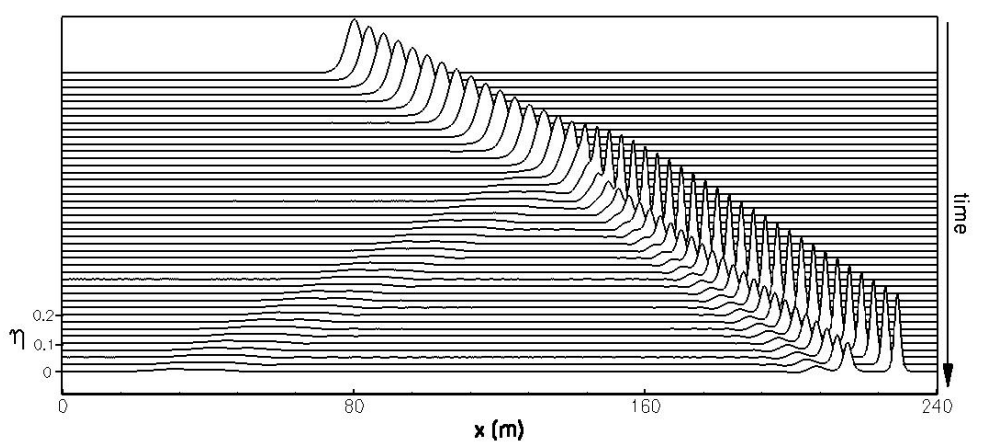

Figure 16: Splitting of a solitary wave propagating over a submerged shelf : numerical computation using SUPG scheme.
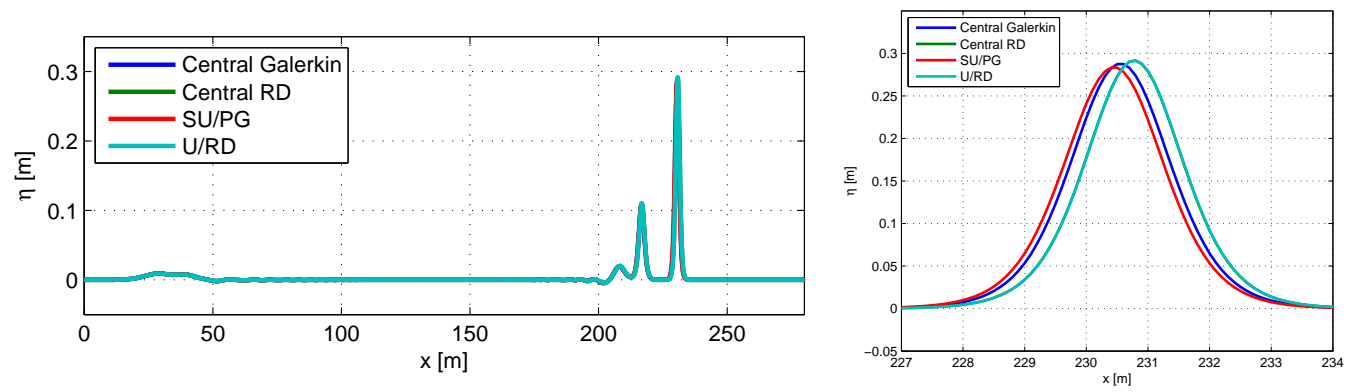

Figure 17: Solitary wave interaction with a shelf. Superposition of the solutions obtained with the cRD, URD, cG and SUPG schemes (left), and close-up of the highest peak region to highlight the difference between the solutions computed (right).

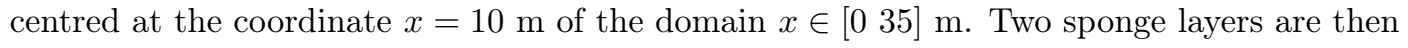
used at the two boundaries of the domain, extended on the regions $x \in\left[\begin{array}{lll}0 & 3\end{array}\right] \mathrm{m}$ on the left and $x \in[3235] \mathrm{m}$ on the right, in order to absorb any wave reaching the boundaries.

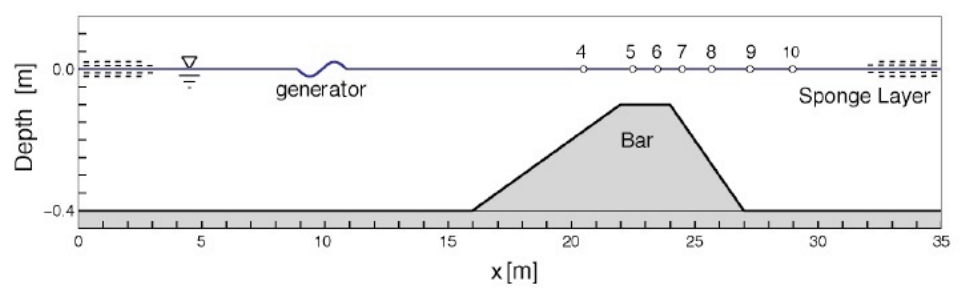

Figure 18: Sketch of the computational configuration of the numerical test of the propagation over a submerged bar.

The values of grid size and time step have been set to $\Delta x=0.04 \mathrm{~m}$ and $\Delta t=0.0323$, similar to those used by other authors (see e.g. [16] and references therein). For this choice of parameters, the cRD scheme has shown a strongly unstable behavior. For all the other schemes, computed results are compared with experimental data in gauges placed at $x_{4}=$ $20.5 \mathrm{~m}, x_{5}=22.5 \mathrm{~m}, x_{6}=23.5 \mathrm{~m}, x_{7}=24.5 \mathrm{~m}, x_{8}=25.7 \mathrm{~m}$, and $x_{9}=27.3 \mathrm{~m}$, denoted as gauge $4,5,6,7,8,9,10$ in the following. Numerical simulations have been run for a over $40 \mathrm{~s}$, and the computed signals in the gauges have been compared to the experimental ones 
after a steady periodic flow has been obtained. Due to the periodic character of the solution, as in [16, 51] a phase calibration has been necessary to be able to compare computed and measured signals. This calibration has been performed here by introducing a small time shift in the computed results, such that the phase error w.r.t. the data in gauge 4 is minimized, as shown on figure 19. Once this time shift is obtained, the same value is applied to all the other gauges.

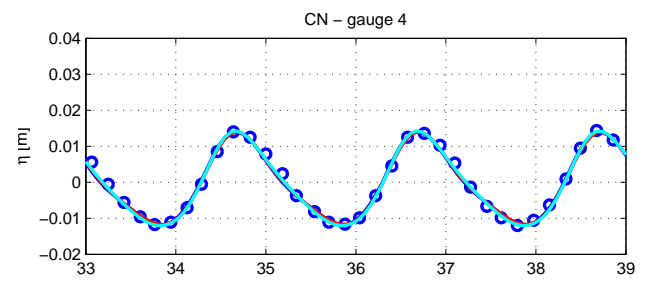

Figure 19: Time evolution at the gauges coordinates of the surface elevation of sinusoidal wave encountering a submerged bar, with initial amplitude $A=0.01 \mathrm{~m}$ and period $T=2.02$ s. Results in gauge 4 used for phase calibration w.r.t experiments of [47].

The comparison between computed and experimental signals is reported on figure 20 . The schemes performed very well for all the gauges up to the crest of the bar (gauge 8), maintaining a good agreement with the experimental results and showing only a weak error in the phase shift despite the strong nonlinearity. It should be noted that part of the discrepancies between the laboratory data and the models results might be due to the experimental setup.

As the waves pass the back slope of the bar, some discrepancies with the data appear, especially at the section of gauge 10. This might be due to the activation of a numerical phenomenon which decoupled the higher harmonics from the primary longer wave; these are released as free waves propagating generating inaccuracy. The upwind residual distribution scheme appears to be the most sensible to this kind of problem.

This problem provides a severe test of the model as nonlinearity initially steepens the waves on the up-slope and then the increasing depth behind the bar decomposes the waves into short wave components. This resulted in a rapidly varying profile behind the bar with the exact form depending crucially on the dispersive characteristics of the numerical model. As expected from the theoretical limitations of the model used, some discrepancies show up as higher harmonics are released behind the bar. Nevertheless the agreement is still very reasonable, and the discretization schemes proposed represent quite accurately the nonlinear and dispersive properties of the original continuous model. Moreover the similar resolution properties of the SUPG and cG schemes are once more confirmed.

\section{Preliminary validation in two space dimensions}

We discuss in this section a preliminary validation of the two-dimensional variant of the model. In particular, we will discuss the form of the equations chosen $\mathrm{n}$ two space, dimension and the extension, implementation and validation of the SUPG scheme on tests involving wave diffraction on variable bathymetries. The objective of this section is to show the potential of the upwind stabilized SUPG scheme, which has been retained as the best between the two upwind discretizations discussed, in accurately predicting complex wave transformation patterns in two space dimensions.

\subsection{Enhanced Boussinesq equations in two space dimensions}

In two space dimensions, we have adopted the form of the enhanced Boussinesq equations proposed in [48, and also used in [40] as a basis for a mixed Galerkin finite element approximation. Denoting by $\vec{q}$ the mass flux vector, $\vec{q} \equiv\left(q_{x}, q_{y}\right)$, with $\vec{u} \equiv\left(u_{x}, u_{y}\right)$ the velocity 

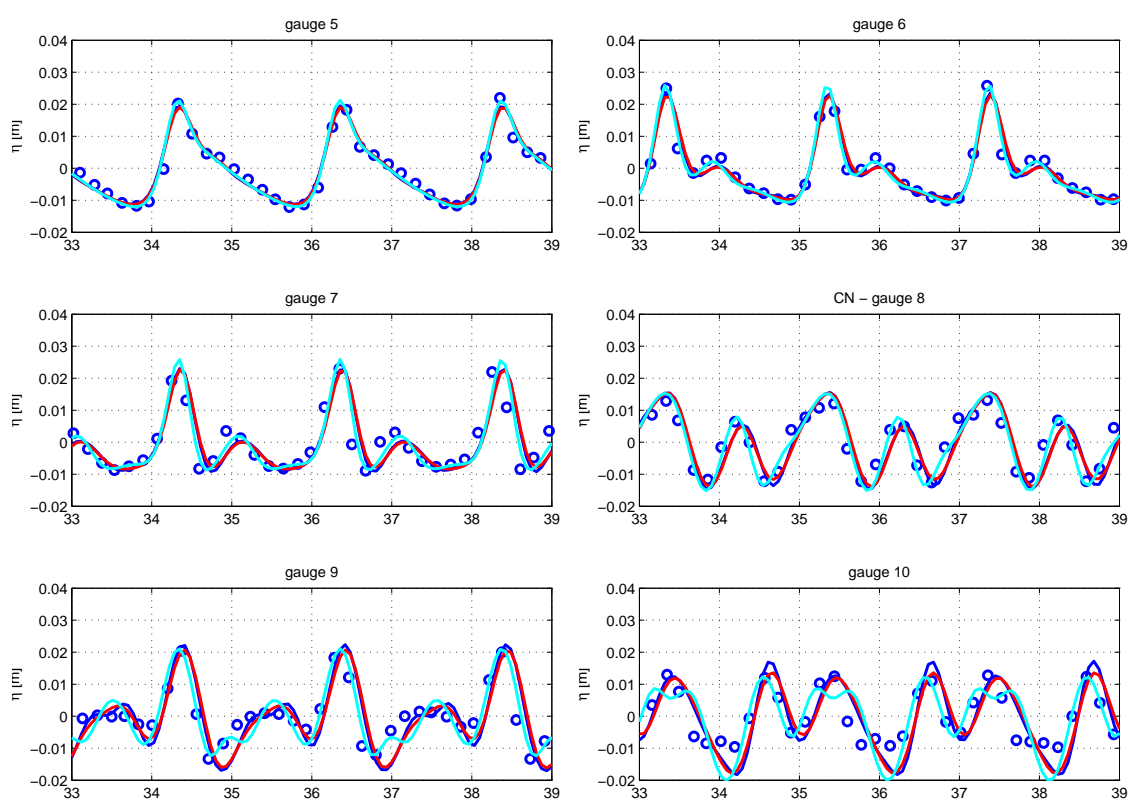

Figure 20: Time evolution at the gauges coordinates of the surface elevation of sinusoidal wave encountering a submerged bar, with initial amplitude $A=0.01 \mathrm{~m}$ and period $T=2.02$ s. In figure, o represents the experimental data,- is the cG, - is the SUPG and - is the URD scheme.

vector, and with the same notation used in one dimension for the other variables, the model equations read :

$$
\left\{\begin{array}{l}
\partial_{t} \eta+\nabla \cdot \vec{q}=0 \\
\partial_{t} q+\nabla \cdot(\vec{u} \otimes \vec{q})+g H \nabla \eta+\vec{\psi}=0
\end{array}\right.
$$

where $\vec{\psi} \equiv\left(\psi_{x}, \psi_{y}\right)$ are the dispersive terms of the model which, following [48, 40] are written as

$$
\left\{\begin{array}{l}
\psi_{x}=-B h^{2} \partial_{t x} \nabla \cdot \vec{q}-\frac{1}{6} h \partial_{x} h \partial_{t}\left(\nabla \cdot \vec{q}+\partial_{x} q_{x}\right)-\frac{1}{6} h \partial_{y} h \partial_{t x} q_{y}-\beta g h^{2} \partial_{x} w^{\eta} \\
\psi_{y}=-B h^{2} \partial_{t y} \nabla \cdot \vec{q}-\frac{1}{6} h \partial_{y} h \partial_{t}\left(\nabla \cdot \vec{q}+\partial_{y} q_{y}\right)-\frac{1}{6} h \partial_{x} h \partial_{t y} q_{x}-\beta g h^{2} \partial_{y} w^{\eta} \\
w^{\eta}=\nabla \cdot(h \nabla \eta)
\end{array}\right.
$$

This form of the enhanced model reduces exactly to (1) for constant bathymetry in the one dimensional case, however, for variable bathymetry this corresponds to a variant of the model with improved shoaling coefficient proposed in [48. The main advantage of using this form of the equations, as compared to e.g. the ones used in [50], is that all the higher order derivatives of $\eta$, including the mixed ones, appear through the sole auxiliary variable $w^{\eta}$ (third in (34). This allows to reduce the number of auxiliary variables whose reconstruction is needed in the numerical implementation of the model (cf. next section). The values of the dispersion coefficients $\beta$ and $B$ are set, as in one dimension and as in [48, 40, to $\beta=1 / 15$ and $B=\beta+1 / 3$.

\subsection{SUPG scheme : implementation in two dimensions}

The remaining part of the paper is devoted to the preliminary validation of the SUPG scheme in two space dimensions. If $\Omega$ denotes the spatial domain, we consider an unstructured 
triangulation of the spatial domain $\Omega_{\mathrm{h}}$, of typical size $\mathrm{h}$, and where $\mathrm{K}$ denotes the generic element, while $\mathrm{K}_{i}$ denotes the set of elements containing node $i$. On $\Omega_{\mathrm{h}}$ we consider piecewise linear continuos approximations $\eta_{\mathrm{h}}$ and $\vec{q}_{\mathrm{h}}$ of the variables of type (5), with standard piecewise linear continuous Lagrange bases (cf. figure 21).
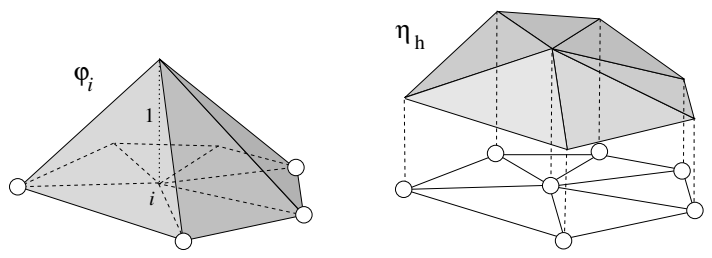

Figure 21: $P^{1}$ finite element interpolation

The discrete version of $(33)$ on $\Omega_{\mathrm{h}}$ in node $i$ will be written for short as

$$
\mathcal{R}_{i}^{\mathrm{cG}}\left(\eta_{\mathrm{h}}, \vec{q}_{\mathrm{h}}\right)+\sum_{\mathrm{K} \in \Omega_{h h}} \int_{\mathrm{K}} \mathcal{A} \cdot \nabla \varphi_{i} \tau_{\mathrm{K}} r_{\mathrm{h}}^{\mathrm{MS}}=0
$$

where, as in one dimension, $\mathcal{R}_{i}^{\mathrm{cG}}$ represents the nodal equation obtained with the Galerkin scheme, while in the streamline upwind term we have introduced $\mathcal{A} \equiv\left(A_{x}, A_{y}\right)$ with $A_{x}$ and $A_{y}$ the Jacobians of $x$ and $y$ components of the conservative fluxes of the two-dimensional NLSW equations. We recall that $\forall \vec{\xi} \in \mathbb{R}^{2}$ the matrix $\mathcal{A}_{\vec{\xi}}=\mathcal{A} \cdot \vec{\xi}$ admits a full set of linearly independent eigenvectors associated to the thee eigenvalues $\vec{u} \cdot \vec{\xi}$ and $\vec{u} \cdot \vec{\xi} \pm C\|\vec{\xi}\|$ with $C^{2}=g H$. We refer to [46] and references therein for detailed expressions of these quantities.

In two space dimensions, the $\mathrm{cG}$ term $\mathcal{R}_{i}^{\mathrm{cG}}$ has been implemented as

$$
\mathcal{R}_{i}^{\mathrm{cG}}=\mathcal{R}_{i}^{\mathrm{cG}-\mathrm{NLSW}}+\mathcal{R}_{i}^{\mathrm{cG}-\psi}
$$

where, as in the one dimensional case, the first term can be written after re-integrating by-parts the Galerkin statement as

$$
\mathcal{R}_{i}^{\mathrm{cG}-\mathrm{NLSW}}=\left[\begin{array}{l}
\int_{\Omega_{\mathrm{h}}} \varphi_{i}\left(\partial_{t} \eta_{\mathrm{h}}+\nabla \cdot \vec{q}_{\mathrm{h}}\right) \\
\int_{\Omega_{\mathrm{h}}} \varphi_{i}\left(\partial_{t} \vec{q}_{\mathrm{h}}+\nabla \cdot(\vec{u} \otimes \vec{q})_{\mathrm{h}}+g H_{\mathrm{h}} \nabla \eta_{\mathrm{h}}\right)
\end{array}\right]
$$

For the dispersive component we have $\mathcal{R}_{i}^{\mathrm{cG}-\psi}=\left(0, \mathcal{R}_{i}^{\mathrm{cG}-\psi_{x}}, \mathcal{R}_{i}^{\mathrm{cG}-\psi_{y}}\right)^{T}$, where the momentum terms have been implemented as

$$
\begin{aligned}
\mathcal{R}_{i}^{\mathrm{cG}-\psi_{x}} & =\int_{\Omega_{h}} B h^{2}\left(\partial_{t x} q_{x \mathrm{~h}} \partial_{x} \varphi_{i}+\frac{1}{2}\left(\partial_{x} q_{y \mathrm{~h}} \partial_{y} \varphi_{i}+\partial_{y} q_{y \mathrm{~h}} \partial_{x} \varphi_{i}\right)\right) \\
& -\frac{1}{6} \int_{\Omega_{\mathrm{h}}} \varphi_{i} h\left(\partial_{x} h \partial_{t}\left(\nabla \cdot \vec{q}_{\mathrm{h}}+\partial_{x} q_{x \mathrm{~h}}\right)+\partial_{y} h \partial_{t x} q_{y \mathrm{~h}}\right)-\int_{\Omega_{\mathrm{h}}} \beta g h^{2} \partial_{x} w_{\mathrm{h}}^{\eta}
\end{aligned}
$$

Fully discrete expressions are obtained after evaluation of (38) with approximate quadrature over each mesh element, with the hypothesis of piecewise linear variation of all the quantities involved. Note that, for symmetry reasons, the mixed derivative term in the first line of (38) has been expressed as an average of two terms for which the partial derivative in each space direction is passed onto the basis function. The form of the $\mathcal{R}_{i}^{\mathrm{cG}-\psi_{y}}$ term is very similar.

The scheme is completed by the expression of the streamline upwind term actually implemented, and by the definition of the nodal equations for the auxiliary variables. Concerning the streamline dissipation term, proceeding as in one dimension (cf. section §3.4, details 
omitted for brevity), we have used the following expression consistent with a $P^{1}$ approximation in space and with a one point linearization of the $N L S W$ Jacobian (cf. equation (16)) :

$$
\int_{\mathrm{K}} \mathcal{A} \cdot \nabla \varphi_{i} \tau_{\mathrm{K}} r_{\mathrm{h}}^{\mathrm{M}} \approx \mathcal{A}_{\mathrm{K}} \cdot \nabla \varphi_{i}^{\mathrm{K}} \tau_{\mathrm{K}} \Phi^{\mathrm{K}}
$$

where in two space dimensions, the element residual $\Phi^{\mathrm{K}}=\left[\begin{array}{cc}\Phi_{\eta}^{\mathrm{K}} & \Phi_{\vec{q}}^{\mathrm{K}}\end{array}\right]^{T}$ is computed as (cf. section $\S 3.2$, equation (8) and $(10)$ )

$$
\begin{aligned}
& \Phi_{\eta}^{\mathrm{K}}=\int_{K}\left(\partial_{t} \eta_{\mathrm{h}}+\nabla \cdot \vec{q}_{\mathrm{h}}\right) \\
& \Phi_{\vec{q}}^{\mathrm{K}}=\int_{K}\left(\partial_{t} \vec{q}_{\mathrm{h}}+\nabla \cdot(\vec{u} \otimes \vec{q})_{\mathrm{h}}+g H_{\mathrm{h}} \nabla \eta_{\mathrm{h}}\right)+\Phi_{\vec{\psi}}^{\mathrm{K}} \\
& \Phi_{\vec{\psi}}^{\mathrm{K}}=\left(\Phi_{\psi_{x}}^{\mathrm{K}}, \Phi_{\psi_{y}}^{\mathrm{K}}\right)^{T}
\end{aligned}
$$

where the dispersive components of the element residual have been coded as

$$
\begin{aligned}
\Phi_{\psi_{x}}^{\mathrm{K}}=-B \int_{\mathrm{K}} & h^{2}\left(\partial_{t x} w_{x \mathrm{~h}}^{q_{x}}+\frac{1}{2}\left(\partial_{t x} w_{y \mathrm{~h}}^{q_{y}}+\partial_{t y} w_{x \mathrm{~h}}^{q_{y}}\right)\right) \\
& -\frac{1}{6} \int_{\mathrm{K}} h\left(\partial_{x} h\left(2 \partial_{t x} q_{x \mathrm{~h}}+\partial_{t y} q_{y \mathrm{~h}}\right)+\partial_{y} h \partial_{t x} q_{y \mathrm{~h}}\right)-\beta g \int_{\mathrm{K}} h^{2} \partial_{x} w_{\mathrm{h}}^{\eta}
\end{aligned}
$$

with auxiliary variables $\vec{w}^{q_{x}} \equiv\left(w_{x}^{q_{x}}, w_{y}^{q_{x}}\right)$ and $\vec{w}^{q_{y}} \equiv\left(w_{x}^{q_{y}}, w_{y}^{q_{y}}\right)$ defined as

$$
\vec{w}^{q_{x}}=\nabla q_{x}, \quad \vec{w}^{q_{y}}=\nabla q_{y}
$$

Note that in (41), as in the Galerkin term (38), the second order mixed derivatives of $q_{y}$ have been implemented, for symmetry reasons, as an average of the $x$ and $y$ derivatives of the components of $\vec{w}^{q_{y}}$. A similar expression is used for $\Phi_{\psi_{y}}^{\mathrm{K}}$. As in one dimension, fully discrete quantities are obtained by evaluating the integrals with numerical quadrature with the hypothesis of linear variation of all the quantities involved.

Lastly, we have implemented the stabilization parameter $\tau_{\mathrm{K}}$ has proposed in [9, 19, 1, 46] (cf equation (17) section $§ 3.4$ )

$$
\tau_{\mathrm{K}}=\left(\sum_{j \in \mathrm{K}}\left|\mathcal{A}_{\mathrm{K}} \cdot \nabla \varphi_{j}\right|\right)^{-1}
$$

while the nodal equations for the auxiliary variables $w_{\mathrm{h}}^{\eta}, \vec{w}^{q_{x}}$, and $\vec{w}^{q_{y}}$ have been implemented following [2, 3, 43] as

$$
\begin{aligned}
w_{i}^{\eta} & =-\frac{1}{\sum_{\mathrm{K} \in \mathrm{K}_{i}}|K|} \sum_{\mathrm{K} \in \mathrm{K}_{i}} \int_{\mathrm{K}} h \nabla \eta_{\mathrm{h}} \cdot \nabla \varphi_{i} \\
\vec{w}_{i}^{q_{x}} & =\frac{1}{\sum_{\mathrm{K} \in \mathrm{K}_{i}}|K|} \sum_{\mathrm{K} \in \mathrm{K}_{i}}|K| \nabla q_{x}^{\mathrm{K}} \\
\vec{w}_{i}^{q_{y}} & =\frac{1}{\sum_{\mathrm{K} \in \mathrm{K}_{i}}|K|} \sum_{\mathrm{K} \in \mathrm{K}_{i}}|K| \nabla q_{y}^{\mathrm{K}}
\end{aligned}
$$


where, while all of the above expressions can be obtained by an $L^{2}$ projection with mass lumping $\mathrm{n}$ the left hand side, the last two boil down to a Green-Gauss reconstruction of the nodal gradients of $\vec{q}[3,43]$.

As in one dimension, the numerical simulations presented in the next sections make use of Crank-Nicholson integration in time, obtained by simply replacing the time derivatives $\partial_{t}(\cdot)$ by time increments $\Delta^{n+1}(\cdot)=(\cdot)^{n+1}-(\cdot)^{n}$, and evaluating the remaining quantities at the $n+1 / 2$ level (cf. section $\S 5$ ). The Newton algorithm discussed in section $\S 5$ has been implemented also in two dimensions, the Jacobian matrix of the $\eta$ and $\vec{q}$ algebraic equations having been evaluated analytically, including the non-local terms arising from the reconstruction of the auxiliary variables. In the tests presented, we have made use of the additional reflective boundary condition

$$
\vec{q}_{\mathrm{h}} \cdot \vec{n}=0, \nabla \eta_{\mathrm{h}} \cdot \vec{n}=0
$$

which has been implemented in a strong form by modifying the two components of the right had side of the $\vec{q}_{\mathrm{h}}$ equations (and the related Jacobian entries) $\mathcal{R}_{i}^{\vec{q}}$ such that

$$
\mathcal{R}_{i}^{\vec{q}} \cdot \vec{n}=0
$$

at each Newton iteration.

\subsection{Wave diffraction over a semi-circular shoal}

This test reproduces one of the tests carried out in [53] involving the study of the focusing effect induced by a semicircular shoal on wave trains of different periods. The experiments were carried out in a wave tank $6.096 \mathrm{~m}$ wide and $25.6 \mathrm{~m}$ long, its middle portion consisted in a semi-circular shoal leading the water depth to decrease from $h_{0}=0.4572 \mathrm{~m}$ (at the wavemaker) to $0.1524 \mathrm{~m}$ at the end of the tank. The bottom topography is described by the equation

$$
z= \begin{cases}0 & \text { if } \quad 0 \leq x<10.67-G(y) \\ (10.67-G(y)-x) / 25 & \text { if } 10.67-G(y) \leq x<18.29-G(y) \\ 0.30480 & \text { if } 10.29-G(y) \leq x\end{cases}
$$

with $G(y)=\sqrt{y(6.096-y)}$. The depth $h$ is obtained as $h=h_{0}-z$. A contour plot of the bathymetry is given on figure 22 .

Three sets of experiments are reported in 53 corresponding to wave periods $T=1.0 \mathrm{~s}$, $2.0 \mathrm{~s}$ and $3.0 \mathrm{~s}$, and for each period two or three different wave amplitudes were considered. Free surface elevation measurements were taken along the tank centerline, and an harmonic analysis of the time dependent signals was performed to obtain the amplitude of frequency components. This experiment has become a typical test to verify the capabilities of a model to reproduce nonlinear refraction and diffraction (see e.g. [50, 35, 41, 40, 10] to cite a few).

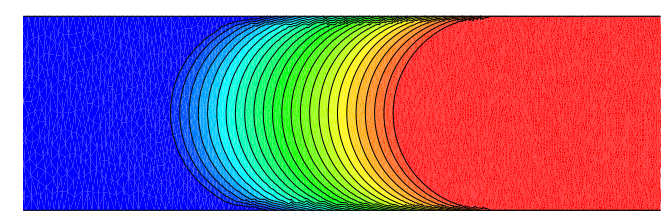

Figure 22: Wave diffraction over a semi-circular shoal : bathymetry contours.

We consider here the case with period $T=2 \mathrm{~s}$ and amplitude $A=0.0075 \mathrm{~m}$. The computational domain is the rectangle $[-10,36] m \times[0,6.096] \mathrm{m}$. Periodic waves are generated 
by means of the internal generator described in section $\S 6.3$, centered at $x=-2 \mathrm{~m}$. Sponge layers (cf. section §6.1) of a length of $5 \mathrm{~m}$ are set at the left and right ends of the domain, while reflective boundary conditions are imposed along the top and bottom boundaries.
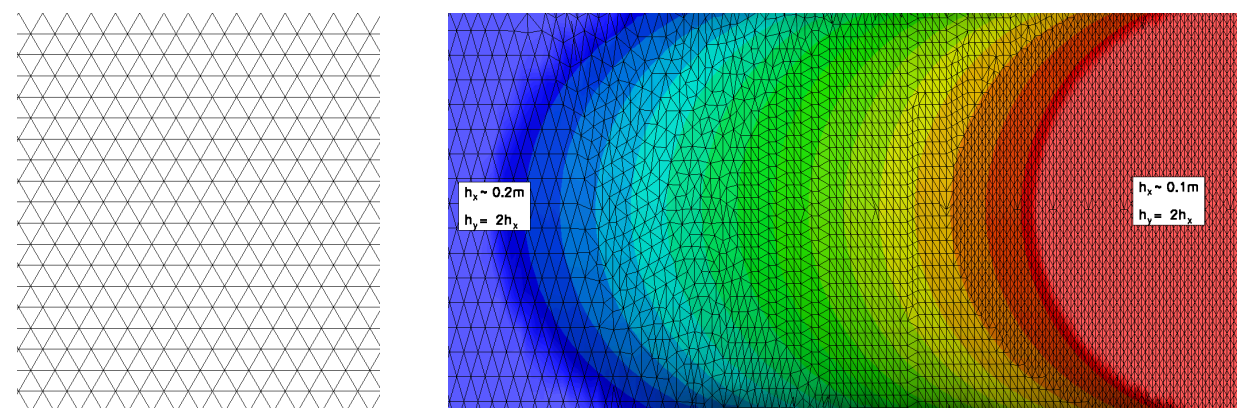

Figure 23: Wave diffraction over a semi-circular : close-up of the structured (left) and unstructured (right) grids.

We compare the solution obtained with the SUPG on two triangulation of the spatial domain. The first is a uniform grid composed of diagonally cut quadrilaterals, as depicted on the left on figure 23. The mesh size in the $x$ direction is uniform and equal to $\mathrm{h}_{x}=0.1 \mathrm{~m}$. The second mesh is an unstructured triangulation with $\mathrm{h}_{x} \approx 0.2 \mathrm{~m}$ at the left and right boundaries. As shown on the right in figure 23, $\mathrm{h}_{x}$ is progressively reduced when approaching the shoal from the left end boundary to reach the value o $\mathrm{h}_{x} \approx 0.1 \mathrm{~m}$ before the end of the shoal. For both grids, the mesh size in the $y$ direction always verifies $\mathrm{h}_{y}=2 \mathrm{~h}_{x}$. Computations have been run for $100 \mathrm{~s}$ with $\Delta t \approx 0.03$. The data along the centerline has been extracted in the last $25 \mathrm{~s}$ of the simulations and analyzed by means of DFT.
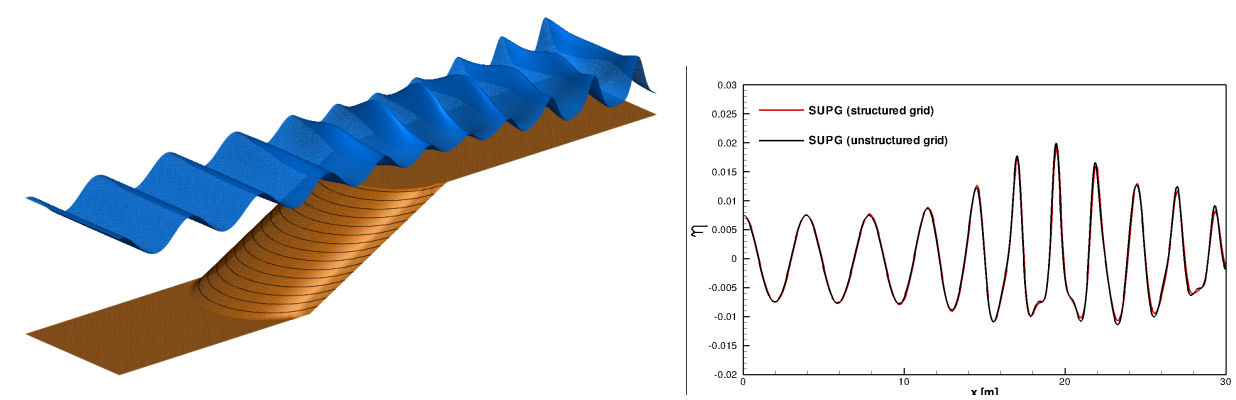

Figure 24: Wave diffraction over a semi-circular : exaggerated 3D view of the free surface (left) and comparison of the corresponding structured and unstructured computed free surface level along the centerline $y=3.048 \mathrm{~m}$ (right) grids.

Concerning the solution obtained, we first report a view of the free surface level when the peak of the periodic wave enters the domain on the left on figure 24. showing the nice capturing of the wave transformation over the shoal. The differences between structured and unstructured grid results are very hard to see. As a example, we report on the right on figure 24 the centerline distribution of the wave height corresponding to the left picture's solution. Clearly, the two results only present minor differences, which is highly encouraging since the unstructured mesh only contains half the number of elements (30705 triangles instead of 64470).

Lastly, on figure 25 we analyze the DFT of the time dependent data extracted along centerline. The left picture shows the comparison of the computed wave amplitudes for the first three harmonics with the experimental data of [53]. The agreement between computed and measured amplitudes is very good. The slight over-prediction of the amplitudes, as well as the behavior at the end of the wave tank is comparable to what is found by other authors (see e.g. [50, 35, 41, 40, 10]). On the right picture on figure 25 we compare the DFT of 

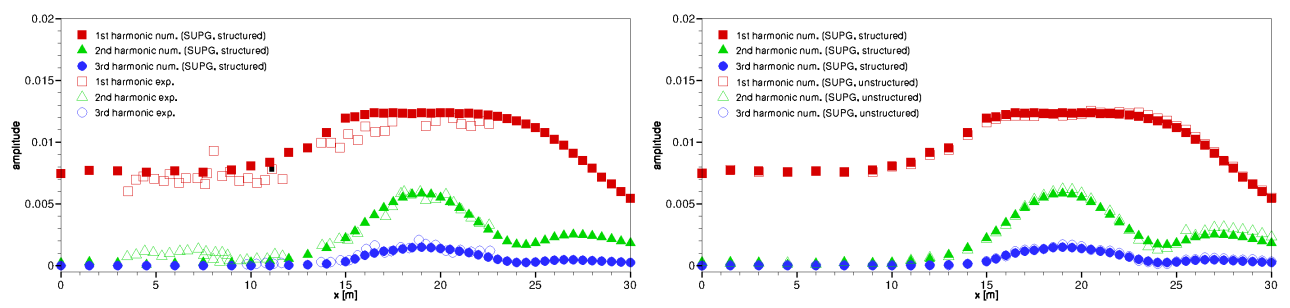

Figure 25: Wave diffraction over a semi-circular : DFT of the centerline data from the structured grid results compared to experimental data of [53. (left) and comparison of the harmonic content on the centerline data from the structured and unstructured grid results.

the structured and unstructured grid results. The comparison shows again that only minor differences between the two results are present. These are mainly visible in a better resolution of the higher harmonics, perhaps due to a slight over-refinement of the mesh, w.r.t. the target minimum meshsize of $0.1 \mathrm{~m}$.

\subsection{Wave diffraction over an elliptic shoal}

This test reproduces the experiment of [12] studying the refraction and diffraction of monochromatic waves over a complex bathymetry. This is a standard test to verify models based on the mild-slope equations but it is often also used as a test for extended Boussinesq models (see e.g. and [50, 52, 51, 25] references therein). A sketch of the experiment is reported on the left picture on figure 26. The actual wave tank is $20 \mathrm{~m}$ wide and $22 \mathrm{~m}$ long. The bathymetry consists of an elliptic shoal mounted on a ramp of constant slope, forming a $20^{\circ}$ angle with the $x$ axis. The maximum water depth is $h_{0}=0.45 \mathrm{~m}$ at the wave maker, while the bathymetry is given by the formula $z=z_{0}+z_{s}$, where

$$
\begin{aligned}
& z_{0}= \begin{cases}\left(5.82+y_{r}\right) / 50 & \text { if } y_{r} \leq-5.82 \\
0 & \text { otherwise }\end{cases} \\
& z_{s}= \begin{cases}-0.3+\frac{1}{2} \sqrt{1-\left(\frac{x_{r}}{5}\right)^{2}-\left(\frac{4 y_{r}}{15}\right)^{2}} & \text { if }\left(\frac{x_{r}}{4}\right)^{2}+\left(\frac{y_{r}}{3}\right)^{2} \leq 1 \\
0 & \text { otherwise }\end{cases}
\end{aligned}
$$

where the transformed coordinates $\left(x_{r}, y_{r}\right)$ are defined as

$$
x_{r}=x \cos \left(20^{\circ}\right)-y \sin \left(20^{\circ}\right), \quad y_{r}=x \sin \left(20^{\circ}\right)+y \cos \left(20^{\circ}\right)
$$

The water depth is computed as $h=h_{0}-z$. The incoming periodic wave has period $T=1 \mathrm{~s}$, and amplitude $A=0.0232 \mathrm{~m}$. In [12], the wave elevation was measured in 8 different sections along which the normalized time average wave height distribution has been computed. Here we will report comparison with the data along the three sections drawn on the left picture on figure 26, which correspond to sections $2,3,5$, and 7 of the experiment of [12].

The computational domain is the square $[-10,10] \mathrm{m} \times[-17,15] \mathrm{m}$. The incoming periodic wave is obtained by means of the internal wave generator described in section $\S 6.3$, centered at $y=-13 \mathrm{~m}$, while sponge layers of $4 \mathrm{~m}$ thickness are placed at the bottom and top ends of the domain. As in [50, reflective boundary conditions are imposed on the left and right boundaries. The simulation has been run on an unstructured grid. The grid has been refined around the region of the interaction. In particular, the gridsize in the $y$ direction varies from $\mathrm{h}_{y} \approx 0.1$ on the bottom and top boundaries to $\mathrm{h}_{y} \approx 0.05$ in the region around the shoal where the interaction takes place. In the $x$ direction, the gridsize is $\mathrm{h}_{x}=2 \mathrm{~h}_{y}$. A close up view of the mesh refinement in the bottom left region of the computational domain is reported in the right picture on figure 26. Computations have been run until time $t=50 \mathrm{~s}$ with $\Delta t \approx 0.02 \mathrm{~s}$. 

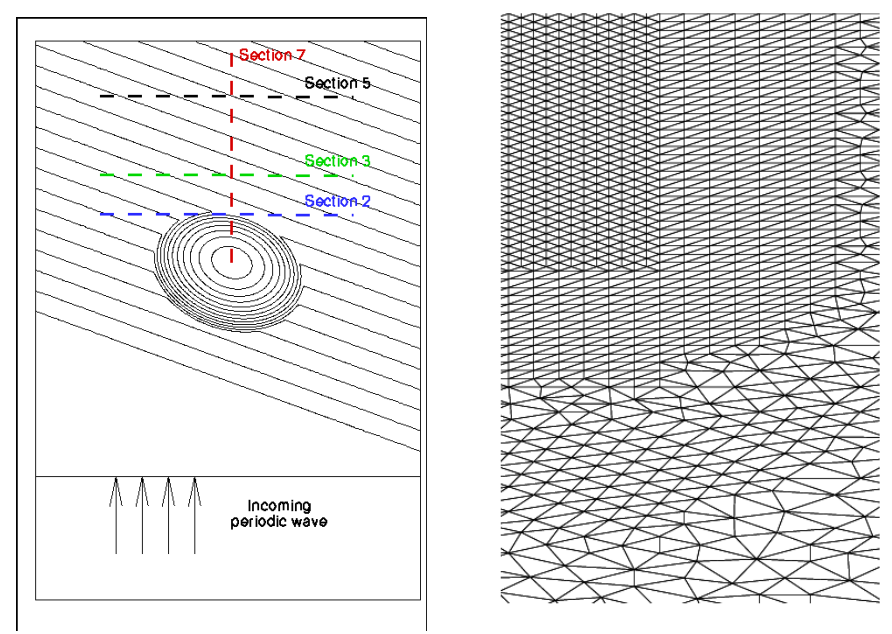

Figure 26: Wave diffraction over an elliptic shoal. Left : sketch of the problem with bathymetry contours and position of the experimental sections. Right : close up view of the mesh.

Three dimensional visualizations of the water elevation computed at time $t=50 \mathrm{~s}$ are reported on figure 27. The left picture shows the region of the interaction. Both pictures provide a visualization of the complex pattern obtained from the diffraction of the incoming periodic wave on the elliptic shoal. To compare our results with the experiments of [12] time dependent data have been extracted from $t=25 \mathrm{~s}$ to $t=50 \mathrm{~s}$ from the sections (cf. leftmost picture on figure 26 and [12]) : section $2 \equiv\{y=3 m \mid-5 m \leq x \leq 5 m\}$, section $3 \equiv\{y=5 m \mid-5 m \leq x \leq 5 m\}$, section $5 \equiv\{y=9 m \mid-5 m \leq x \leq 5 m\}$, section $7 \equiv$ $\{x=0 m \mid 0 m \leq y \leq 10 m\}$. The data obtained has been analyzed using the zero up-crossing principle to isolate single waves and compute the average wave height distributions, the wave height of a single wave being defined as $\eta_{\max }-\eta_{\min }$. The results, normalized by the incoming wave amplitude $A=0.0232 \mathrm{~m}$, are reported on figure 28 .
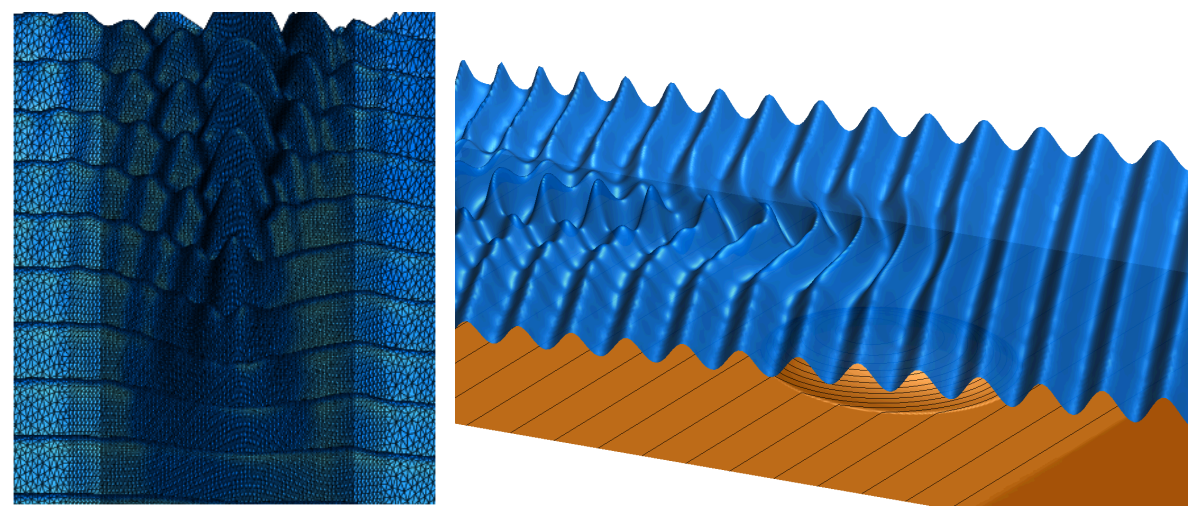

Figure 27: Wave diffraction over an elliptic shoal, results at $t=50 \mathrm{~s}$. Left : top view of the free surface with mesh. Right : exaggerated 3D view of the free surface with bathymetry.

The agreement of our computational results with the experiments is very satisfactory, and compares very favorably with what can be found in published literature (see e.g. [50, 52] and references therein). 

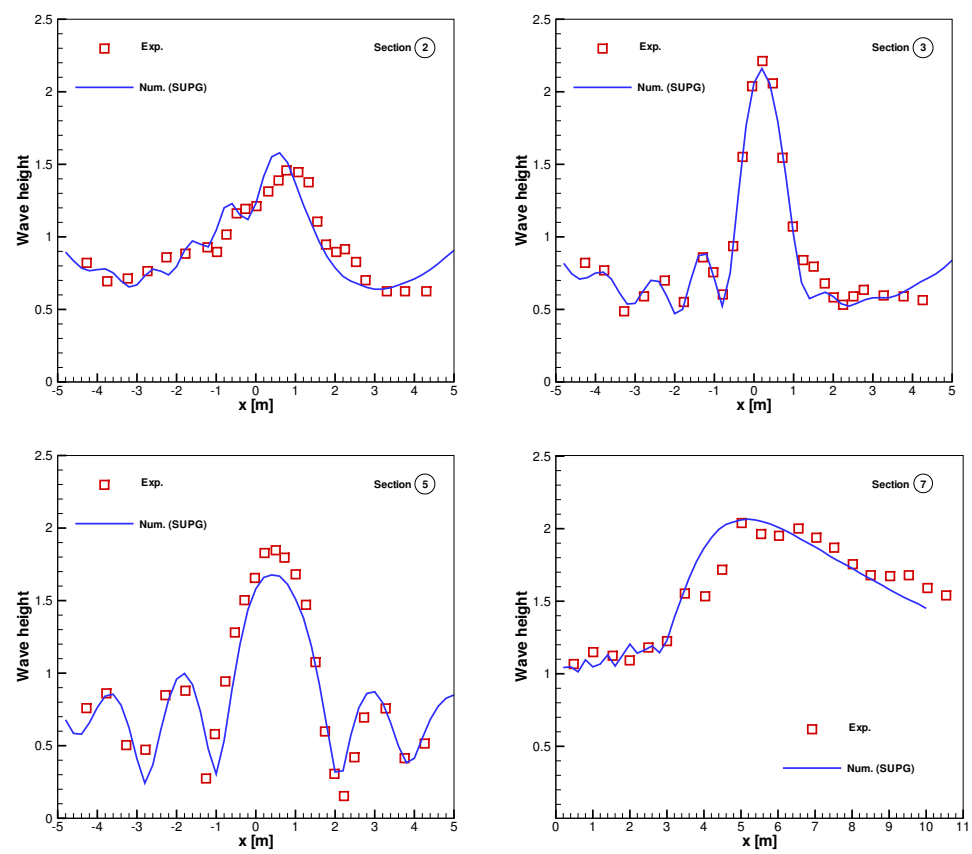

Figure 28: Wave diffraction over an elliptic shoal. Comparison of the computed average wave height with the experimental data [12] in sections $2,3,5$, and 7 .

\section{Conclusions and future work}

In this paper we have presented an investigation of the applicability of upwind stabilized schemes of the residual distribution and continuous Galerkin type for the approximation of the enhanced Boussinesq equations of [41, 48]. We have in particular given one possible formulation of upwind residual distribution, or URD, and Streamline Upwind stabilized continuous Galerkin, or SUPG, for this system of equations, and analyzed in one space dimension the truncation and dispersion error properties of the schemes. The analysis shows that, while the URD scheme, as well as its centered counterpart, have formal accuracy close to that of the standard second order centered finite difference scheme, the SUPG scheme proposed has, at least on structured grids, better accuracy, showing a formal truncation error on the free surface level of third order, and a remarkably low dispersion error, which is even smaller than that of the fourth order centered finite difference scheme.

We have given a detailed description of the implementation of the schemes, including the generation of exact solitary waves for the enhanced Boussinesq equations of Madsen and Sørensen, following the approach of [33], and the generation of periodic waves by means of an internal wave generator. The numerical results obtained in one space dimension have confirmed the higher accuracy of the SUPG and continuous Galerkin scheme, compared to the $\mathrm{RD}$ ones, and shown a nice capturing of the physics of solitary and periodic wave propagation and transformation over variable bathymetries.

The conclusion of the one dimensional tests being that the SUPG has accuracy very close to that of the unstabilized continuous Galerkin scheme, we have discussed the extension and preliminary validation of the two-dimensional version of the SUPG. The results obtained on unstructured triangulations are indeed quite promising, showing a nice capturing of the multidimensional physics of wave propagation.

Future work will involve both model and scheme improvements. From the modeling point of view, we plan to introduce wave breaking by locally reverting to the NLSW equations, allowing breaking wave fronts to become shocks. To do that, we will introduce local nonlin- 
ear discontinuity capturing techniques either based on nonlinear residual distribution type positivity preserving limited schemes [46], or on more standard finite element discontinuity capturing operators [28, 31].

Another development will involve the extension of our study to genuinely nonlinear models of the Green-Naghdi type, such as the one proposed in [36, 14]

Scheme development will proceed in several directions. One of these is certainly the study of the influence of different time stepping choices on the accuracy of the model. So far in our experience, the inner Newton loop has shown remarkably good convergence, provided that a good approximation of the Jacobian is at hand. This indicates that, due to the presence of mixed space-time derivatives, implicit high order schemes, or explicit multistep ones, might be competitive with multistage explicit schemes, provided that the proper accuracy can be guaranteed. Standard high order implicit finite diference, space time, and explicit multistep schemes will be studied in terms of dispersion error. Lastly, higher order spatial interpolation is a must to allow lower accuracy on grids with a small number of elements. We will in particular consider two lines of research : the use of hierarchical bases eventually coupled to local variations of the degree of the polynomial [54,5], and the use of high order non-Lagrange bases of the such as NURBS or Bezier [30, 4].

\section{A Dissipation and dispersion parameters}

We report here the finite difference expressions of all the schemes analyzed in section $\S 4$ when applied to the linearized equations (2), including their discrete dispersion parameters. All the derivation is done by assuming an equally spaced mesh of size $\mathrm{h}=\Delta x, C_{0}^{2}=g h_{0}$ denotes the square of the linearized Shallow Water celerity, while the notation $\mu_{\Delta x}=k \Delta x$ has been used in the expressions of the dispersion parameters.

\section{A.1 Second order central finite differences}

The second order centered finite difference scheme applied to system (2) yields

$$
\begin{aligned}
& \frac{d \eta_{i}}{d t}+\frac{h_{0}}{2 \Delta x}\left(u_{i+1}-u_{i-1}\right)=0 \\
& \frac{d u_{i}}{d t}-\frac{B h_{0}^{2}}{\Delta x^{2}}\left(\frac{d u_{i-1}}{d t}-2 \frac{d u_{i}}{d t}+\frac{d u_{i+1}}{d t}\right) \\
& +\frac{g}{2 \Delta x}\left(\eta_{i+1}-\eta_{i-1}\right)-\beta \frac{g h_{0}^{2}}{2 \Delta x^{3}}\left(-\eta_{i-2}+2 \eta_{i-1}-2 \eta_{i+1}+\eta_{i+2}\right)=0
\end{aligned}
$$

A lengthy calculation leads to the truncation error :

$$
\begin{aligned}
\mathrm{TE}_{F D 2}^{\eta}= & -\frac{h_{0} \Delta x^{2}}{6} \partial_{x^{3}} u_{i}-\frac{h_{0} \Delta x^{4}}{120} \partial_{x^{5}} u_{i}+\mathscr{O}\left(\Delta x^{6}\right) \\
\mathrm{TE}_{F D 2}^{u}= & -\frac{\Delta x^{2}}{6} \partial_{x^{2}}\left(-\frac{B h_{0}^{2}}{2} \partial_{x^{2} t} u_{i}+g \partial_{x} \eta_{i}-\frac{7}{5} \beta_{i} g h_{0}^{2} \partial_{x^{3}} \eta_{i}\right)+ \\
& -\frac{\Delta x^{4}}{40} \partial_{x^{4}}\left(-\frac{B h_{0}^{2}}{9} \partial_{x^{2} t} u_{i}+\frac{g}{3} \partial_{x} \eta_{i}-\beta_{i} g h_{0}^{2} \partial_{x^{3}} \eta_{i}\right)+ \\
& +\mathscr{O}\left(\Delta x^{6}\right)
\end{aligned}
$$

Replacing in the scheme the time dependent nodal values by a Fourier mode in space, we obtain for the discrete dispersion parameters the following expressions :

$$
\begin{aligned}
\xi_{\mathrm{FD} 2} & =0 \\
\omega_{\mathrm{FD} 2}^{2} & =\frac{N_{\mathcal{F D} 2}}{D_{\mathcal{F D} 2}}
\end{aligned}
$$


where

$$
\begin{aligned}
& N_{\mathrm{FD} 2}=C_{0}^{2} k^{2} \frac{\sin ^{2} \mu_{\Delta x}}{\mu_{\Delta x}^{2}}+2 \beta g h_{0}^{3} k^{4} \frac{\sin ^{2} \mu_{\Delta x}}{\mu_{\Delta x}^{4}}\left(1-\cos \mu_{\Delta x}\right) \\
& D_{\mathrm{FD} 2}=1+2 B \frac{h_{0}^{2} k^{2}}{\mu_{\Delta x}^{2}}\left(1-\cos \mu_{\Delta x}\right)
\end{aligned}
$$

\section{A.2 Fourth order central finite differences}

The fourth order centered finite difference scheme applied to system (2) yields

$$
\begin{aligned}
& \frac{d \eta_{i}}{d t}+\frac{h_{0}}{12 \Delta x}\left(u_{i-2}-8 u_{i-1}+8 u_{i+1}-u_{i+2}\right)=0 \\
& \quad \frac{d u_{i}}{d t}-\frac{B h_{0}^{2}}{12 \Delta x^{2}}\left(-\frac{d u_{i-2}}{d t}+16 \frac{d u_{i-1}}{d t}-30 \frac{d u_{i}}{d t}+16 \frac{d u_{i+1}}{d t}-\frac{d u_{i+2}}{d t}\right) \\
& +\frac{g}{12 \Delta x}\left(\eta_{i-2}-8 \eta_{i-1}+8 \eta_{i+1}-\eta_{i+2}\right) \\
& -\beta \frac{g h_{0}^{2}}{8 \Delta x^{3}}\left(\eta_{i-3}-8 \eta_{i-2}+13 \eta_{i-1}-13 \eta_{i+1}+8 \eta_{i+2}-\eta_{i+3}\right)=0
\end{aligned}
$$

A lengthy calculation allows to compute the truncation error estimate :

$$
\begin{aligned}
\mathrm{TE}_{F D 4}^{\eta} & =\frac{h_{0} \Delta x^{4}}{30} \partial_{x^{5}} u_{i}+\mathscr{O}\left(\Delta x^{6}\right) \\
\mathrm{TE}_{F D 4}^{u} & =\Delta x^{4} \partial_{x^{4}}\left(-\frac{2}{135} B h_{0}^{2} \partial_{x^{2} t} u_{i}+\frac{g}{30} \partial_{x} \eta_{i}-\frac{653}{10080} \beta_{i} g h_{0}^{2} \partial_{x^{3}} \eta_{i}\right)+\mathscr{O}\left(\Delta x^{6}\right)
\end{aligned}
$$

Replacing in the scheme the time dependent nodal values by a Fourier mode in space, we obtain for the discrete dispersion parameters the following expressions :

$$
\begin{aligned}
\xi_{\mathrm{FD} 4} & =0 \\
\omega_{\mathrm{FD} 4}^{2} & =\frac{N_{\mathcal{F D} 4}}{D_{\mathcal{F} \mathcal{D} 4}}
\end{aligned}
$$

where

$$
\begin{aligned}
N_{\mathrm{FD} 4}= & \frac{C_{0}^{2} k^{2}}{36 \mu_{\Delta x}}\left(8 \sin \mu_{\Delta x}-\sin 2 \mu_{\Delta x}\right)^{2}+ \\
& +\beta \frac{h_{0}^{2} k^{2}}{\mu_{\Delta x}^{2}}\left(\sin 3 \mu_{\Delta x}-8 \sin 2 \mu_{\Delta x}+13 \sin \mu_{\Delta x}\right) \\
D_{\mathrm{FD} 4}= & 1+B \frac{h_{0}^{2} k^{2}}{6 \mu_{\Delta x}^{2}}\left(15-16 \cos \mu_{\Delta x}+\cos 2 \mu_{\Delta x}\right)
\end{aligned}
$$

\section{A.3 Continuous Galerkin scheme}

When applied to system (2) on a uniform mesh with definition 22 of the auxiliary variable $w_{i}^{\eta}$ yielding

$$
w_{i}^{\eta}=\frac{\eta_{i+1}-2 \eta_{i}+\eta_{i-1}}{\Delta x^{2}}
$$

$\mathrm{RR} \mathrm{n}^{\circ} 8311$ 
the continuous Galerkin scheme (7) reduces to

$$
\begin{aligned}
\frac{\Delta x}{6} \frac{d \eta_{i-1}}{d t}+ & \frac{2 \Delta x}{3} \frac{d \eta_{i}}{d t}+\frac{\Delta x}{6} \frac{d \eta_{i+1}}{d t}+\frac{h_{0}}{2}\left(u_{i+1}-u_{i-1}\right)=0 \\
\frac{\Delta x}{6} \frac{d u_{i-1}}{d t}+ & \frac{2 \Delta x}{3} \frac{d u_{i}}{d t}+\frac{\Delta x}{6} \frac{d u_{i+1}}{d t}+\frac{g}{2}\left(\eta_{i+1}-\eta_{i-1}\right)-\frac{B h_{0}^{2}}{\Delta x}\left(\frac{d u_{i-1}}{d t}-2 \frac{d u_{i}}{d t}+\frac{d u_{i+1}}{d t}\right) \\
- & \beta \frac{g h_{0}^{2}}{2 \Delta x^{2}}\left(-\eta_{i-2}+2 \eta_{i-1}-2 \eta_{i+1}+\eta_{i+2}\right)=0
\end{aligned}
$$

Note that the only difference w.r.t the second order finite difference scheme is the presence of a non-diagonal mass matrix multiplying the $\partial_{t} \eta$ and $\partial_{t} u$ terms, otherwise the two schemes are identical. A long calculation leads to the expression of the truncation error

$$
\begin{aligned}
\mathrm{TE}_{\mathrm{cG}}^{\eta}= & -\frac{\Delta x^{4}}{24} \partial_{x^{4}}\left(\frac{1}{3} \partial_{t} u_{i}+\frac{h_{0}}{5} \partial_{x} u_{i}\right)+\mathscr{O}\left(\Delta x^{6}\right) \\
\mathrm{TE}_{\mathrm{cG}}^{u}= & -\frac{\Delta x^{2}}{2} \partial_{x^{2}}\left(\frac{1}{3} \partial_{t} u_{i}-\frac{B h_{0}^{2}}{6} \partial_{x^{2} t} u_{i}+\frac{1}{3} g \partial_{x} \eta_{i}-\beta g h_{0}^{2} \partial_{x^{3}} \eta_{i}\right)+ \\
& -\frac{\Delta x^{4}}{8} \partial_{x^{4}}\left(\frac{1}{9} \partial_{t} u_{i}-\frac{B h_{0}^{2}}{45} \partial_{x^{2} t} u_{i}+\frac{g}{15} \partial_{x} \eta_{i}-\frac{1}{5} \beta g h_{0}^{2} \partial_{x^{3}} \eta_{i}\right)+ \\
& +\mathscr{O}\left(\Delta x^{6}\right)
\end{aligned}
$$

Replacing in the scheme the time dependent nodal values by a Fourier mode in space, we obtain for the discrete dispersion parameters the following expressions :

$$
\begin{aligned}
& \xi_{\mathrm{cG}}=0 \\
& \omega_{\mathrm{cG}}^{2}=C_{0}^{2} k^{2} \frac{\sin ^{2}\left(\mu_{\Delta x}\right)}{\mu^{2}} \frac{1+2 \beta \mu^{2} \frac{1-\cos \left(\mu_{\Delta x}\right)}{\mu_{\Delta x}^{2}}}{\frac{2+\cos \left(\mu_{\Delta x}\right)}{3}\left(\frac{2+\cos \left(\mu_{\Delta x}\right)}{3}+2 B \mu^{2} \frac{1-\cos \left(\mu_{\Delta x}\right)}{\mu_{\Delta x}^{2}}\right)}
\end{aligned}
$$

\section{A.4 Centered residual distribution}

When applied to system (2), definitions 22 of $w^{\eta}$ yields

$$
w_{i}^{\eta}=\frac{\eta_{i+1}-2 \eta_{i}+\eta_{i-1}}{\Delta x^{2}} \text { and }
$$

while $w^{q}$ is replaced by a $w^{u} \approx \partial_{x} u$ similarly defined, which under the present hypotheses is computed as

$$
w_{i}^{u}=\frac{u_{i+1}-u_{i-1}}{2 \Delta x}
$$

the centered residual distribution scheme reduces to

$$
\begin{aligned}
& \frac{\Delta x}{4} \frac{d \eta_{i-1}}{d t}+ \frac{\Delta x}{2} \frac{d \eta_{i}}{d t}+\frac{\Delta x}{4} \frac{d \eta_{i+1}}{d t}+\frac{h_{0}}{2}\left(u_{i+1}-u_{i-1}\right)=0 \\
& \frac{\Delta x}{4} \frac{d u_{i-1}}{d t}+\frac{\Delta x}{2} \frac{d u_{i}}{d t}+\frac{\Delta x}{4} \frac{d u_{i+1}}{d t}+\frac{g}{2}\left(\eta_{i+1}-\eta_{i-1}\right)-\frac{B h_{0}^{2}}{2 \Delta x}\left(\frac{d u_{i-2}}{d t}-2 \frac{d u_{i}}{d t}+\frac{d u_{i+2}}{d t}\right) \\
&-\beta \frac{g h_{0}^{2}}{2 \Delta x^{2}}\left(-\eta_{i-2}+2 \eta_{i-1}-2 \eta_{i+1}+\eta_{i+2}\right)=0
\end{aligned}
$$

Note that the difference w.r.t the second order finite difference scheme is the presence of a non-diagonal mass matrix multiplying the $\partial_{t} \eta$ and $\partial_{t} u$ terms, and the fact that the second 
order derivative of $u$ in space is approximated with a larger stencil. A long calculation shows that the truncation error can be written as

$$
\begin{aligned}
\mathrm{TE}_{\mathrm{cRD}}^{\eta}= & \frac{\Delta x^{2}}{2} \partial_{x^{2}}\left(\frac{1}{2} \partial_{t} u_{i}+\frac{h_{0}}{3} \partial_{x} u_{i}\right)+\mathscr{O}\left(\Delta x^{4}\right) \\
\mathrm{TE}_{\mathrm{cRD}}^{u}= & \Delta x^{2} \partial_{x^{2}}\left(\frac{1}{4} \partial_{t} u_{i}-\frac{1}{3} \frac{B h_{0}^{2}}{2} \partial_{x^{2} t} u_{i}+\frac{1}{6} g \partial_{x} \eta_{i}-\frac{1}{4} \beta g h_{0}^{2} \partial_{x^{3}} \eta_{i}\right)+ \\
& +\mathscr{O}\left(\Delta x^{4}\right)
\end{aligned}
$$

Replacing the time dependent nodal values by a Fourier mode in space, we obtain for the discrete dispersion parameters the following expressions :

$$
\begin{aligned}
& \xi_{\mathrm{cRD}}=0 \\
& \omega_{\mathrm{cRD}}^{2}=C_{0}^{2} k^{2} \frac{\sin ^{2}\left(\mu_{\Delta x}\right)}{\mu^{2}} \frac{1+2 \beta \mu^{2} \frac{1-\cos \left(\mu_{\Delta x}\right)}{\mu_{\Delta x}^{2}}}{\frac{1+\cos \left(\mu_{\Delta x}\right)}{2}\left(\frac{1+\cos \left(\mu_{\Delta x}\right)}{2}+\frac{B \mu^{2}}{2} \frac{1-\cos \left(2 \mu_{\Delta x}\right)}{\mu_{\Delta x}^{2}}\right)}
\end{aligned}
$$

\section{A.5 SUPG scheme}

In the linearized case, the matrix associated to the Shallow Water quasi-linear form is

$$
A_{0}=\left[\begin{array}{cc}
0 & h_{0} \\
g & 0
\end{array}\right]
$$

Straightforward eigenvalue decomposition show that the sign of this matrix can be written as

$$
\operatorname{sign}\left(A_{0}\right)=\left[\begin{array}{cc}
0 & C_{0} / g \\
g / C_{0} & 0
\end{array}\right]
$$

Using this expression, and the pointwise expressions of the auxiliary variables recalled above, we can deduce the form of the SUPG scheme (18) applied to system (2) :

$$
\begin{gathered}
\frac{\Delta x}{6} \frac{d \eta_{i-1}}{d t}+\frac{2 \Delta x}{3} \frac{d \eta_{i}}{d t}+\frac{\Delta x}{6} \frac{d \eta_{i+1}}{d t}+\frac{h_{0}}{2}\left(u_{i+1}-u_{i-1}\right)-\frac{C_{0}}{2 g}\left\{\frac{\Delta x}{2}\left(\frac{d u_{i+1}}{d t}-\frac{d u_{i-1}}{d t}\right)\right. \\
-\frac{B h_{0}^{2}}{\Delta x}\left(\frac{d u_{i+2}}{d t}-2 \frac{d u_{i+1}}{d t}+2 \frac{d u_{i-1}}{d t}-\frac{d u_{i-2}}{d t}\right)-g\left(\eta_{i+1}-2 \eta_{i}+\eta_{i-1}\right) \\
\left.-\frac{\beta g h_{0}^{2}}{\Delta x^{2}}\left(\eta_{i+2}-4 \eta_{i+1}+6 \eta_{i}-4 \eta_{i-1}+\eta_{i-2}\right)\right\}=0
\end{gathered}
$$

$$
\frac{\Delta x}{6} \frac{d u_{i-1}}{d t}+\frac{2 \Delta x}{3} \frac{d u_{i}}{d t}+\frac{\Delta x}{6} \frac{d u_{i+1}}{d t}+\frac{g}{2}\left(\eta_{i+1}-\eta_{i-1}\right)-\frac{B h_{0}^{2}}{\Delta x}\left(\frac{d u_{i-1}}{d t}-2 \frac{d u_{i}}{d t}+\frac{d u_{i+1}}{d t}\right)
$$

$\mathrm{RR} \mathrm{n}^{\circ} 8311$

$$
\begin{aligned}
& -\beta \frac{g h_{0}^{2}}{2 \Delta x^{2}}\left(-\eta_{i-2}+2 \eta_{i-1}-2 \eta_{i+1}+\eta_{i+2}\right) \\
& -\frac{g}{2 \Gamma}\left\{\frac{\Delta x}{2}\left(\frac{d \eta_{i+1}}{d t}-\frac{d \eta_{i-1}}{d t}\right)+h_{0}\left(u_{i+1}-2 u_{i}+u_{i-1}\right)\right\}=0
\end{aligned}
$$


A long calculation leads to the expression of the truncation error

$$
\begin{aligned}
\mathrm{TE}_{\mathrm{SUPG}}^{\eta}= & \frac{C_{0} \Delta x^{3}}{2 g} \partial_{x^{3}}\left(\frac{1}{3} \partial_{t} u_{i}-\frac{1}{2} B h_{0}^{2} \partial_{x^{2} t} u_{i}+\frac{1}{6} g \partial_{x} \eta_{i}-\frac{1}{3} \beta g h_{0}^{2} \partial_{x^{3}} \eta_{i}\right)+ \\
& -\frac{\Delta x^{4}}{24} \partial_{x^{4}}\left(\frac{1}{3} \partial_{t} u_{i}+\frac{h_{0}}{5} \partial_{x} u_{i}\right)+\mathscr{O}\left(\Delta x^{5}\right) \\
\mathrm{TE}_{\mathrm{SUPG}}^{u}= & -\frac{\Delta x^{2}}{2} \partial_{x^{2}}\left(\frac{1}{3} \partial_{t} u_{i}-\frac{B h_{0}^{2}}{6} \partial_{x^{2} t} u_{i}+\frac{1}{3} g \partial_{x} \eta_{i}-\beta g h_{0}^{2} \partial_{x^{3}} \eta_{i}\right)+ \\
& +\frac{g \Delta x^{3}}{6 C_{0}} \partial_{x}\left(\partial_{t} \eta_{i}+\frac{h_{0}}{2} \partial_{x} u_{i}\right)+ \\
& -\frac{\Delta x^{4}}{8} \partial_{x^{4}}\left(\frac{1}{9} \partial_{t} u_{i}-\frac{B h_{0}^{2}}{45} \partial_{x^{2} t} u_{i}+\frac{g}{15} \partial_{x} \eta_{i}-\frac{1}{5} \beta g h_{0}^{2} \partial_{x^{3}} \eta_{i}\right)+ \\
& +\mathscr{O}\left(\Delta x^{5}\right)
\end{aligned}
$$

Replacing the time dependent nodal values by a Fourier mode in space, we obtain for the discrete dispersion parameters the following expressions :

$$
\begin{aligned}
& \xi_{\mathrm{SUPG}}=-\frac{A_{\nu}}{2 A_{\nu^{2}}} \\
& \omega_{\mathrm{SUPG}}^{2}=\frac{A_{s}}{A_{\nu^{2}}}-\xi^{2}
\end{aligned}
$$

where

$$
\begin{aligned}
A_{\nu^{2}}= & \frac{\left(2+\cos \mu_{\Delta x}\right)}{3}\left(\frac{\left(2+\cos \mu_{\Delta x}\right)}{3}+2 B \mu^{2} \frac{\left(1-\cos \left(\mu_{\Delta x}\right)\right)}{\mu_{\Delta x}^{2}}\right)+ \\
& +\frac{\sin ^{2} \mu_{\Delta x}}{4}\left(1+2 B \mu^{2} \frac{\left(1-\cos \mu_{\Delta x}\right)}{\mu_{\Delta x}^{2}}\right) ; \\
A_{\nu}= & C_{0} k \frac{\left(1-\cos \mu_{\Delta x}\right)}{\mu_{\Delta x}}\left(\frac{\left(2+\cos \mu_{\Delta x}\right)}{3}+2 B \mu^{2} \frac{\left(1-\cos \left(\mu_{\Delta x}\right)\right)}{\mu_{\Delta x}^{2}}\right)+ \\
& +2 \beta C_{0} \mu^{2} \frac{\left(1-\cos \mu_{\Delta x}\right)^{2}}{\mu_{\Delta x}^{3}}\left(\frac{\left(2+\cos \mu_{\Delta x}\right)}{3}+2 B \mu^{2} \frac{\left(1-\cos \left(\mu_{\Delta x}\right)\right)}{\mu_{\Delta x}^{2}}\right)+ \\
& -C_{0} k \frac{\sin ^{2} \mu_{\Delta x}}{2 \mu_{\Delta x}}\left(1+2 B \mu^{2} \frac{\left(1-\cos \mu_{\Delta x}\right)}{\mu_{\Delta x}^{2}}\right)\left(1+2 \beta \mu^{2} \frac{\left(1-\cos \mu_{\Delta x}\right)}{\mu_{\Delta x}^{2}}\right)+ \\
& +C_{0} k \frac{\left(1-\cos \mu_{\Delta x}\right)^{2}}{6 \mu_{\Delta x}} ; \\
A_{s}= & C_{0}^{2} k^{2} \frac{\left(1-\cos \mu_{\Delta x}\right)^{2}}{\mu_{\Delta x}^{2}}\left(1+2 \beta \mu^{2} \frac{\left(1-\cos \mu_{\Delta x}\right)}{\mu_{\Delta x^{2}}}\right)+ \\
& +C_{0}^{2} k^{2} \frac{\sin ^{2} \mu_{\Delta x}}{\mu_{\Delta x}^{2}}\left(1+2 B \mu^{2} \frac{\left(1-\cos \mu_{\Delta x}\right)}{\mu_{\Delta x}^{2}}\right) .
\end{aligned}
$$

\section{A.6 Upwind residual distribution}

Using the expressions of the sign of the Shallow Water Jacobian in the linearized case, and the expressions of the auxiliary variables recalled above, the upwind RD scheme applied to 
the linearized system (2) becomes

$$
\begin{gathered}
\frac{\Delta x}{4} \frac{d \eta_{i-1}}{d t}+\frac{\Delta x}{2} \frac{d \eta_{i}}{d t}+\frac{\Delta x}{4} \frac{d \eta_{i+1}}{d t}+\frac{h_{0}}{2}\left(u_{i+1}-u_{i-1}\right)-\frac{C_{0}}{2 g}\left\{\frac{\Delta x}{2}\left(\frac{d u_{i+1}}{d t}-\frac{d u_{i-1}}{d t}\right)\right. \\
-\frac{B h_{0}^{2}}{\Delta x}\left(\frac{d u_{i+2}}{d t}-2 \frac{d u_{i+1}}{d t}+2 \frac{d u_{i-1}}{d t}-\frac{d u_{i-2}}{d t}\right)-g\left(\eta_{i+1}-2 \eta_{i}+\eta_{i-1}\right) \\
\left.-\frac{\beta g h_{0}^{2}}{\Delta x^{2}}\left(\eta_{i+2}-4 \eta_{i+1}+6 \eta_{i}-4 \eta_{i-1}+\eta_{i-2}\right)\right\}=0 \\
\begin{array}{r}
\frac{\Delta x}{4} \frac{d u_{i-1}}{d t}+\frac{\Delta x}{2} \frac{d u_{i}}{d t}+\frac{\Delta x}{4} \frac{d u_{i+1}}{d t}+\frac{g}{2}\left(\eta_{i+1}-\eta_{i-1}\right)-\frac{B h_{0}^{2}}{2 \Delta x}\left(\frac{d u_{i-2}}{d t}-2 \frac{d u_{i}}{d t}+\frac{d u_{i+2}}{d t}\right) \\
-\beta \frac{g h_{0}^{2}}{2 \Delta x^{2}}\left(-\eta_{i-2}+2 \eta_{i-1}-2 \eta_{i+1}+\eta_{i+2}\right) \\
-\frac{g}{2 C_{0}}\left\{\frac{\Delta x}{2}\left(\frac{d \eta_{i+1}}{d t}-\frac{d \eta_{i-1}}{d t}\right)+h_{0}\left(u_{i+1}-2 u_{i}+u_{i-1}\right)\right\}=0
\end{array}
\end{gathered}
$$

A long calculation leads to the truncation error :

$$
\begin{aligned}
\mathrm{TE}_{\mathrm{URD}}^{\eta} & =-\frac{\Delta x^{2}}{2} \partial_{x^{2}}\left(\frac{1}{2} \partial_{t} u_{i}+\frac{h_{0}}{3} \partial_{x} u_{i}\right)+ \\
& +\frac{C_{0} \Delta x^{3}}{2 g} \partial_{x^{3}}\left(\frac{1}{3} \partial_{t} u_{i}-\frac{1}{2} B h_{0}^{2} \partial_{x^{2} t} u_{i}+\frac{1}{6} g \partial_{x} \eta_{i}-\frac{1}{3} \beta g h_{0}^{2} \partial_{x^{3}} \eta_{i}\right)+ \\
& -\frac{\Delta x^{4}}{24} \partial_{x^{4}}\left(\frac{1}{2} \partial_{t} u_{i}+\frac{h_{0}}{5} \partial_{x} u_{i}\right)+\mathscr{O}\left(\Delta x^{5}\right) \\
\mathrm{TE}_{\mathrm{URD}}^{u} & =-\Delta x^{2} \partial_{x^{2}}\left(\frac{1}{4} \partial_{t} u_{i}-\frac{1}{3} \frac{B h_{0}^{2}}{2} \partial_{x^{2} t} u_{i}+\frac{1}{6} g \partial_{x} \eta_{i}-\frac{1}{4} \beta g h_{0}^{2} \partial_{x^{3}} \eta_{i}\right)+ \\
& +\frac{g \Delta x^{3}}{6 C_{0}} \partial_{x}\left(\partial_{t} \eta_{i}+\frac{h_{0}}{2} \partial_{x} u_{i}\right)+ \\
& -\frac{\Delta x^{4}}{4} \partial_{x^{4}}\left(\frac{1}{3} \partial_{t} u_{i}-\frac{8}{45} B h_{0}^{2} \partial_{x^{2} t} u_{i}+\frac{g}{30} \partial_{x} \eta_{i}-\frac{1}{10} \beta g h_{0}^{2} \partial_{x^{3}} \eta_{i}\right)+ \\
& +\mathscr{O}\left(\Delta x^{5}\right)
\end{aligned}
$$

Replacing the time dependent nodal values by a Fourier mode in space, we obtain for the discrete dispersion parameters the following expressions :

$\mathrm{RR} \mathrm{n}^{\circ} 8311$

$$
\begin{aligned}
& \xi_{\mathrm{URD}}=-\frac{A_{\nu}}{2 A_{\nu^{2}}} \\
& \omega_{\mathrm{URD}}^{2}=\frac{A_{s}}{A_{\nu^{2}}}-\xi^{2}
\end{aligned}
$$


where

$$
\begin{aligned}
& A_{\nu^{2}}= \frac{\left(1+\cos \mu_{\Delta x}\right)}{2}\left(\frac{\left(1+\cos \mu_{\Delta x}\right)}{2}+B \mu^{2} \frac{\left(1-\cos \left(2 \mu_{\Delta x}\right)\right)}{2 \mu_{\Delta x}^{2}}\right)+ \\
&+\frac{\sin ^{2} \mu_{\Delta x}}{4}\left(1+2 B \mu^{2} \frac{\left(1-\cos \mu_{\Delta x}\right)}{\mu_{\Delta x}^{2}}\right) ; \\
& A_{\nu}= C_{0} k \frac{\left(1-\cos \mu_{\Delta x}\right)}{\mu_{\Delta x}}\left(\frac{\left(1+\cos \mu_{\Delta x}\right)}{2}+B \mu^{2} \frac{\left(1-\cos \left(2 \mu_{\Delta x}\right)\right)}{2 \mu_{\Delta x}^{2}}\right)+ \\
&+2 \beta C_{0} \mu^{2} \frac{\left(1-\cos \mu_{\Delta x}\right)^{2}}{\mu_{\Delta x}^{3}}\left(\frac{\left(1+\cos \mu_{\Delta x}\right)}{2}+B \mu^{2} \frac{\left(1-\cos \left(2 \mu_{\Delta x}\right)\right)}{2 \mu_{\Delta x}^{2}}\right)+ \\
&-C_{0} k \frac{\sin ^{2} \mu_{\Delta x}}{2 \mu_{\Delta x}}\left(1+2 B \mu^{2} \frac{\left(1-\cos \mu_{\Delta x}\right)}{\mu_{\Delta x}^{2}}\right)\left(1+2 \beta \mu^{2} \frac{\left(1-\cos \mu_{\Delta x}\right)}{\mu_{\Delta x}^{2}}\right)^{2} ; \\
& A_{s}=C_{0}^{2} k^{2} \frac{\left(1-\cos _{\mu_{\Delta x}}\right)^{2}}{\mu_{\Delta x}^{2}}\left(1+2 \beta \mu^{2} \frac{\left(1-\cos \mu_{\Delta x}\right)}{\mu_{\Delta x^{2}}}\right)+ \\
&+ C_{0}^{2} k^{2} \frac{\sin ^{2} \mu_{\Delta x}}{\mu_{\Delta x}^{2}}\left(1+2 B \mu^{2} \frac{\left(1-\cos \mu_{\Delta x}\right)}{\mu_{\Delta x}^{2}}\right)
\end{aligned}
$$

\section{References}

[1] R. Abgrall. Essentially non oscillatory residual distribution schemes for hyperbolic problems. J. Comput. Phys, 214(2):773-808, 2006.

[2] R. Abgrall, G. Baurin, A. Krust, D. de Santis, and M. Ricchiuto. Numerical approximation of parabolic problems by residual distribution schemes. International Journal for Numerical Methods in Fluids, 71(9):1191-1206, 2013.

[3] R. Abgrall, D. De Santis, and M. Ricchiuto. Construction of a high order Residual Distribution scheme for complex viscous flows. In Seventh International Conference on Computational Fluid Dynamics (ICCFD7), Big Island, Hawaii, USA, July 2012.

[4] R. Abgrall and J. Trefilik. An example of high order residual distribution scheme using non-lagrange elements. Journal of Scientific Computing, 45:3-25, 2010.

[5] S. Adjerid, M. Aiffa, and J.E. Flaherty. Hierarchical finite element bases for triangular and tetrahedral elements. Computer Methods in Applied Mechanics and Engineering, 190:2925 - 2941, 2001.

[6] Patrick Amestoy, Abdou Guermouche, Jean-Yves L'Excellent, and Stéphane Pralet. Hybrid scheduling for the parallel solution of linear systems. Parallel Computing, 32(2):136156, 2006.

[7] Patrick R. Amestoy, Iain S. Duff, Jean-Yves L'Excellent, and Jacko Koster. A fully asynchronous multifrontal solver using distributed dynamic scheduling. SIAM J. Matrix Anal. Appl., 23(1):15-41, January 2001.

[8] W. El Asmar and O. Nwogu. Finite volume solution of boussinesq-type euations on an unstructured grid. in: J. McKee Smith (ed.), Proc. of the $30^{\text {th }}$ Int. Conf. on Coastal Engineering, San Diego, CA, 2006.

[9] T.J. Barth. Numerical methods for gasdynamic systems on unstructured meshes. In Kröner, Ohlberger, and Rohde, editors, An Introduction to Recent Developments in Theory and Numerics for Conservation Laws, volume 5 of Lecture Notes in Computational Science and Engineering, pages 195-285. Springer-Verlag, Heidelberg, 1998. 
[10] S. Beji and K. Nadaoka. A formal derivation and numerical modeling of the improved boussinesq equations for varying depth. Ocean Engineering, 23, 1996.

[11] M.J. Berger and R.J. Leveque. Adaptive mesh refinement using wave-propagation algorithms for hyperbolic systems. SIAM J. Numer. Anal., 35, 1998.

[12] J.C.W. Berkhoff, N. Booy, and A.C. Radder. Verification of numerical wave propagation models for simple harmonic linear water waves. Coastal Engineering, 6:255-279, 1982.

[13] P. Bonneton. Modeling of periodic wave transformation in the inner surf zone. Ocean Engineering, 34, 2007.

[14] P. Bonneton, F. Chazel, D. Lannes, F. Marche, and M. Tissier. A splitting approach for the fully nonlinear and wakly dispersive green-naghdi model. Journal of Computational Physics, 230, 2011.

[15] P. Brufau and P. Garcia-Navarro. Unsteady free surface flow simulation over complex topography with a multidimensional upwind technique. Journal of Computational Physics, 186(2):503 - 526, 2003.

[16] R. Cienfuegos, E. Barthelemy, and P. Bonneton. A fourth order compact finite volume scheme for fully nonlinear and weakly dispersive boussinesq type equations. parti II: Boundary conditions and validation. Int. J. Numer. Meth. Fluids, 53, 2006.

[17] L. Quartapelle D. Ambrosi. A taylor-galerkin method for simulating nonlinear dispersive water waves. Journal of computational physics, 146:546-569, 1998.

[18] H. Deconinck and M. Ricchiuto. Residual distribution schemes: foundation and analysis. In E. Stein, R. de Borst, and T.J.R. Hughes, editors, Encyclopedia of Computational Mechanics. John Wiley \& Sons, Ltd., 2007. DOI: 10.1002/0470091355.ecm054.

[19] H. Deconinck, M. Ricchiuto, and K. Sermeus. Introduction to residual distribution schemes and stabilized finite elements. VKI LS 2003-05, 33 ${ }^{\text {rd }}$ Computational Fluid dynamics Course, von Karman Institute for Fluid Dynamics, 2003.

[20] D. Dutykh, T. Katsaonis, and D. Mitsotakis. Finite volume schemes for dispersive wave propagation and runup. Journal of Computational Physics, 230, 2011.

[21] A.P. Engsig-Karup. Unstructured nodal dg-fem solution of high order boussinesq type equations. PhD Thesis, Technical University of Denmark, 2006.

[22] A.P. Engsig-Karup, J.S. Hesthaven, H.B. Bingham, and T. Warburton. Dg-fem solution for nonlinear wave-structure interaction using boussinesq-type equations. Coastal Engineering, 55, 2008.

[23] C. Eskilsson and S.J. Sherwin. Spectral/hp discontinuous galerkin methods for modelling 2d boussinsq equations. Journal of Computational Physics, 210, 2006.

[24] D.R. Fuhrman and H.B Bingham. Numerical solution of fully nonlinear and highly dispersive boussinesq equations in two horizontal dimensions. Int. J. Numer. Meth. Fluids, 44, 2004.

[25] J.T. Kirby G. Wei. A time-dependent numerical code for extended boussinesq equations. Journal of Waterway, Port, Coastal, and Ocean Engineering, 120:251-261, 1995.

[26] M.F. Gobbi, J.T. Kirby, and G.E. Wei. A fully nonlinear boussinesq model for surface waves. part 2: Extension to o $(k h)^{4}$. J.Fluid.Mech, 405, 2000.

[27] N. Goutal, M.O. Bristeau, and J. Sainte-Marie. Numerical simulation of a nonhydrostatic shallow water model. ComputersGFluids, 47(1), 2011. 
[28] G. Hauke. A symmetric formulation for computing transient shallow water flows. Computer Methods in Applied Mechanics and Engineering, 163(1-4):111-122, 1998.

[29] T.J.R. Hughes and A. Brook. Streamline upwind Petrov-Galerkin formulations for convection dominated flows with particular emphasis on the incompressible Navier-Stokes equations. Comp. Meth. Appl. Mech. Engrg., 32:199-259, 1982.

[30] T.J.R. Hughes, J.A. Cottrell, and Y. Bazilevs. Isogeometric analysis: Cad, finite elements, nurbs, exact geometry and mesh refinement. Comput. Methods Appl. Mech. Eng., 194(39-41):4135-4195, 2005.

[31] T.J.R. Hughes and M. Mallet. A new finite element formulation for CFD IV: a discontinuity-capturing operator for multidimensional advective-diffusive systems. Comp. Meth. Appl. Mech. Engrg., 58:329-336, 1986.

[32] T.J.R. Hughes, G. Scovazzi, and T. Tezduyar. Stabilized methods for compressible flows. J. Sci. Comp., 43:343-368, 2010.

[33] P.H. Taylor J. Orszaghova, A.G.L. Borthwick. From the paddle to the beach - a bousinesq shallow water numerical wave tank based on madsen and sørensen's equations. Journal of Computational Physics, 231, 328-344, 2012.

[34] M. Kazolea, A.I. Delis, I.K. Nikolos, and C.E. Synolakis. An unstructured finite volume boussinesq model for near shore applications. presented at "Frontiers of Computational Physics : Modeling the Earth system", Boulder (CO), December 2012.

[35] M. Kazolea, A.I. Delis, I.K. Nikolos, and C.E. Synolakis. An unstructured finite volume numerical scheme for extended 2d boussinesq-type equations. Coastal Engineering, 69:42-66, 2012.

[36] D. Lannes and P. Bonneton. Derivation of asymptotic two-dimensional time-dependent equations for surface water wave propagation. Physics of Fluids, 21, 2009. 016601 doi:10.1063/1.3053183.

[37] Y.S. Li, S.X. Liu, Y.X. Yu, and G. Lai. Numerical modelling of boussinesq equations by finite element method. Coastal Engineering, 37, 1999.

[38] H. Nishikawa. A first-order system approach for diffusion equation. i: Second-order residual-distribution schemes. Journal of Computational Physics, 227(1):315 - 352, 2007.

[39] O. Nwogu. An alternative form of the boussinesq equations for near-shore wave propagation. Journal of Waterway, Port, Coastal, and Ocean Engineering, pages 618-638, 1994.

[40] L. Sørensen O.R. Sørensen, H. Shaffer. Boussinesq-type modelling using an unstructured finite element technique. Coastal Engineering, 50, 2004.

[41] O.R. Sørensen P.A. Madsen. A new form of the boussinesq equations with improved linear dispersion characteristics. part 2: a slowing varying bathymetry. Coastal Engineering, 18:183-204, 1992.

[42] D.H. Peregnine. Long waves on a beach. J.Fluid.Mech, 27, 1967.

[43] R. Remi, D. De Santis, and M. Ricchiuto. High order preserving residual distribution schemes for advection-diffusion scalar problems on arbitrary grids. Research Report RR-8157, INRIA, November 2012. submitted to SINUM.

[44] M. Ricchiuto. On the c-property and generalized c-property of residual distribution for the shallow water equations. Journal of Scientific Computing, 48:304-318, 2011. 
[45] M. Ricchiuto, R. Abgrall, and H. Deconinck. Application of conservative residual distribution schemes to the solution of the shallow water equations on unstructured meshes,. J. Comput. Phys., 222:287-331, 2007.

[46] M. Ricchiuto and A. Bollermann. Stabilized residual distribution for shallow water simulations. J. Comput. Phys, 228(4):1071-1115, 2009.

[47] J.A. Battjes S. Beji. Numerical simulations of nonlinear-wave propagation over a bar. Coastal Engineering, 23, 1-16, 1994.

[48] H.A. Schaffer and P.A. Madsen. Further enhancements of boussinesq-type equations. Coastal Engineering, 26:1-14, 1995.

[49] M. Tissier, P. Bonneton, F. Marche, F. Chazel, and D. Lannes. A new approach to handle wave breaking in fully nonlinear boussinesq models. Coastal Engineering, 67, 2012 .

[50] M. Tonelli and M. Petti. Hybrid finite volume - finite difference scheme for $2 \mathrm{dh}$ improved boussinesq equations. Coastal Engineering, 56, 2009.

[51] M.A. Walkley. A numerical method for extended boussinesq shallow-water wave equations. PhD Thesis, University of Leeds, 1999.

[52] M.A. Walkley and M. Berzins. A finite element method for the two-dimensional extended boussinesq equations. Int. J. Numer. Meth Fluids, 39, 2002.

[53] R.W. Whalin. The limit of applicability of linear wave refraction theory in a convergence zone. Res.Rep.H-71-3, USACE,Waterways Expt. Station, Vicksburg, MS, 1971.

[54] C.H. Whiting and K.E. Jansen. A stabilized finite element formulation for the incompressible navier-stokes equations using a hierarchical basis. International Journal for Numerical Methods in Fluids, 35, 2001. 


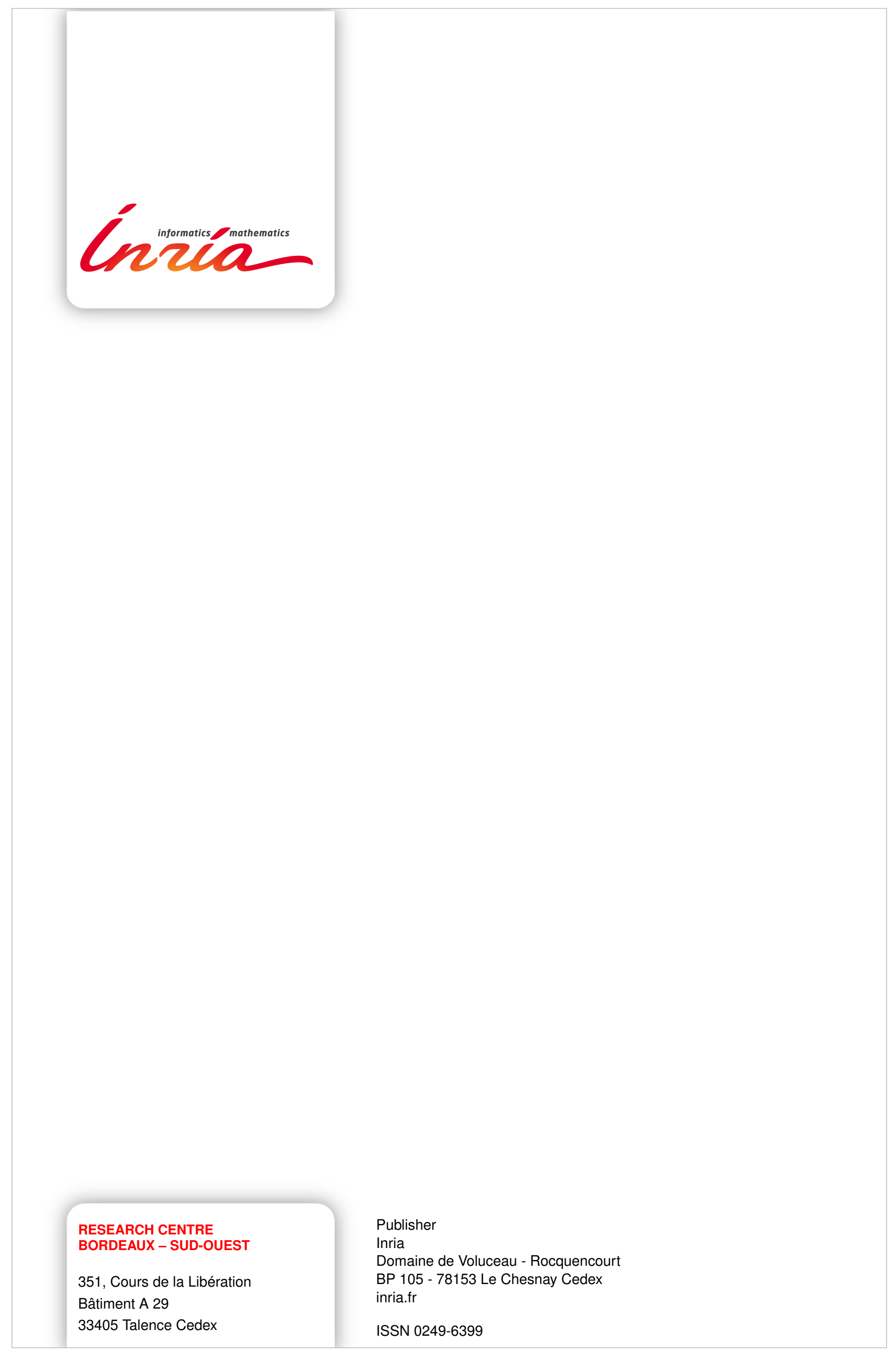

